\title{
Sublytic C5b-9 triggers glomerular mesangial cell apoptosis via XAF1 gene activation mediated by p300-dependent IRF-1 acetylation
}

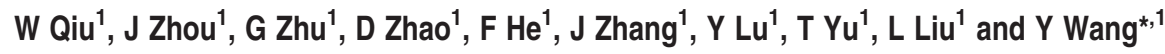

The apoptosis of glomerular mesangial cells (GMCs) in rat Thy-1 nephritis (Thy-1N), a model of human mesangioproliferative glomerulonephritis (MsPGN), is accompanied by sublytic C5b-9 deposition. However, the mechanism by which sublytic C5b-9 induces GMC apoptosis is unclear. In the present studies, the effect of X-linked inhibitor of apoptosis-associated factor 1 (XAF1) expression on GMC apoptosis and the role of p300 and interferon regulatory factor-1 (IRF-1) in mediating XAF1 gene activation were determined, both in the GMCs induced by sublytic C5b-9 (in vitro) and in the renal tissues of rats with Thy-1N (in vivo). The in vitro studies demonstrated that IRF-1-enhanced XAF1 gene activation and its regulation by p300-mediated IRF-1 acetylation were involved in GMC apoptosis induced by sublytic C5b-9. The element of IRF-1 binding to XAF1 promoter and two acetylated sites of IRF-1 protein were also revealed. In vivo, silence of p300, IRF-1 or XAF1 genes in the renal tissues diminished GMC apoptosis and secondary GMC proliferation as well as urinary protein secretion in Thy- $1 \mathrm{~N}$ rats. Together, these data implicate that sublytic C5b-9 induces the expression of both p300 and IRF-1, as well as p300-dependent IRF-1 acetylation that may contribute to XAF1 gene activation and subsequent GMC apoptosis in Thy-1N rats.

Cell Death and Disease (2014) 5, e1176; doi:10.1038/cddis.2014.153; published online 17 April 2014

Subject Category: Immunity

Human mesangioproliferative glomerulonephritis (MsPGN) is a disease characterized by glomerular mesangial cell (GMC) apoptosis and GMC proliferation and extracellular matrix (ECM) secretion. ${ }^{1-4}$ Several studies have found that complement, especially C5b-9 complex, deposits in the glomeruli of patients with MsPGN, ${ }^{5-7}$ leading to $\mathrm{GMC}$ apoptosis and eventual cellular damage. However, the mechanism by which C5b-9 causes GMC injuries remains largely unclear. Rat Thy- 1 nephritis (Thy-1N) is a widely used animal model for human MsPGN. ${ }^{8,9}$ Multiple evidences support the concept that during the process of Thy- $1 \mathrm{~N}$ induction, GMCs undergo apoptosis, necrosis and proliferation. ${ }^{10-13}$ GMC apoptosis in the early phase is considered to be an important contributor to the initiation of Thy- $1 \mathrm{~N}$. $^{10,14}$ Previous studies have confirmed that GMC damage in rat Thy- $1 \mathrm{~N}$ is complement dependent. ${ }^{10,15,16}$ The effects of C5b-9 on nucleated cells are sublytic, which can induce cell apoptosis and other pathologic changes instead of lysis. ${ }^{17-21}$ Nevertheless, the mechanism of GMC apoptosis mediated by sublytic C5b-9 has not yet been well understood.

It is clear that cell apoptosis is associated with the expression of various apoptosis-related genes such as
$X$-linked inhibitor of apoptosis-associated factor 1 $(X A F 1) .{ }^{22,23}$ Reportedly, XAF1 was first identified as an interacting protein of $X$-linked inhibitor of apoptosis (XIAP). It functions as an antagonist of XIAP by rescuing XIAPsuppressed caspase-3 activity, thus promoting cellular apoptosis. ${ }^{24,25}$ XAF1 is also implicated as a tumor suppressor because XAF1 expression is downregulated in a variety of tumor cells including gastric and colon cancer cell lines, and transient expression of XAF1 sensitizes tumor cells to the proapoptotic effects of etoposide. ${ }^{22,23,25}$ Our previous studies demonstrated that the expression of XAF1 was significantly increased in both renal tissues of Thy- $1 \mathrm{~N}$ rats (in vivo) and cultured rat GMCs stimulated with sublytic C5b-9 (in vitro). However, the role of XAF1 gene expression in sublytic C5b-9induced GMC apoptosis of the rats with Thy-1N and its regulation have not been clarified.

Besides, our previous studies also confirmed that the expression of transcription factor interferon regulatory factor-1 (IRF-1) was obviously elevated both in vivo and in vitro, and knockdown of IRF-1 gene suppressed GMC apoptosis upon sublytic C5b-9 stimulation in Thy- $1 \mathrm{~N}$ rats. ${ }^{14}$

\footnotetext{
${ }^{1}$ Department of Microbiology and Immunology, Nanjing Medical University, Nanjing, People's Republic of China

*Corresponding author: Y Wang, Department of Microbiology and Immunology, Nanjing Medical University, 140 Hanzhong Road, Nanjing 210029, People's Republic of China. Tel: + 8625 86862769(L); Fax: +86 25 86508960; E-mail: wangyw1508@njmu.edu.cn

Keywords: Thy-1 nephritis (Thy-1N); glomerular mesangial cell (GMC) apoptosis; sublytic C5b-9 complexes; p300; interferon regulatory factor-1 (IRF-1)

Abbreviations: Ab, antibody; C6DS, complement C6-deficient serum; ChIP, chromatin immunoprecipitation; Co-IP, co-immunoprecipitation; CVF, cobra venom factor; DMEM, Dulbecco's MEM; ECL, enhanced chemiluminescence; ECM, extracellular matrix; EM, electron microscopy; Gadd 45, growth arrest and DNA-damage-inducible 45; GFP, green fluorescence protein; GMC, glomerular mesangial cell; HIS, heat inactive serum; IB, immunoblot; IF, immunofluorescence; IRF-1, interferon regulatory factor-1; IRF-E, IRF-1-binding element; LDH, lactate dehydrogenase; LM, light microscopy; MEM, modified Eagle's medium; MsPGN, mesangioproliferative glomerulonephritis; NHS, normal human serum; NS, normal serum; PCAF, p300/CBP-associated factor; PUMA, p53-upregulated modulator of apoptosis; SD, SpragueDawley; shRNA, short hairpin RNA; Thy-1 Ab, Thy-1 antibody; Thy-1N, Thy-1 nephritis; TUNEL, terminal deoxynucleotidyl transferase-mediated dUTP nick end-labeling; WCE, whole-cell extract; XAF1, X-linked inhibitor of apoptosis-associated factor 1

Received 04.12.13; revised 06.3.14; accepted 10.3.14; Edited by A Stephanou
} 
It is generally known that IRF-1 functions as an important transcription factor of many target genes, including p53upregulated modulator of apoptosis (PUMA) and caspase-8, mainly through binding to IRF-1-binding elements (IRF-Es) in their promoters. ${ }^{14,26}$ Given that JNK1 upregulates XAF1 promoter activity through increasing IRF-1 expression in gastrointestinal cancer, in which IRF-1 binds to IRF-E within XAF1 promoter, ${ }^{27}$ the relationship of $I R F-1$ and $X A F 1$ genes in sublytic C5b-9-induced GMC apoptosis of the rats with Thy-1N, including the activation of XAF1 regulated by IRF-1, needs to be explored.

Mounting evidence suggests that protein acetylation plays important roles in various biological processes including transcriptional regulation. ${ }^{28,29}$ IRF-1 has been shown to be involved in acetylating and activating some transcription factors such as p53 through the recruitment of histone acetyltransferases. ${ }^{30,31}$ It has also been reported that IRF-1 itself is able to undergo acetylation; ${ }^{32,33}$ but the roles of acetylation modification in regulating IRF-1 activation remain unknown. The p300 protein is known as an important transcription coactivator that is able to bind to many transcription factors, such as p53, c-jun and IRF-1, and acetylate these transcription factors, and then regulate cell growth, differentiation and apoptosis. ${ }^{32,34-37}$ Our previous microarray analyses revealed that the expression of transcription coactivator $\mathrm{p} 300$ was obviously upregulated at $3 \mathrm{~h}$ both in vivo (5.9-fold) and in vitro (14.3-fold). However, the role of p300 in mediating IRF-1 acetylation and its ability to promote XAF1 gene activation as well as cellular apoptosis in GMCs triggered by sublytic $\mathrm{C} 5 \mathrm{~b}-9$ in Thy- $1 \mathrm{~N}$ rats need to be elucidated.

In the current study, we not only examined the expression and role of XAF1 gene in sublytic C5b-9-induced GMC apoptosis both in vitro and in vivo, but also determined the synergistic roles of p300 and IRF-1 in regulating XAF1 gene activation in sublytic C5b-9-induced GMCs in Thy- $1 \mathrm{~N}$ rats, including the combination of $\mathrm{p} 300$ and IRF-1 at the protein level, and p300-dependent IRF-1 acetylation.

\section{Results}

Sublytic C5b-9 triggers GMC apoptosis through increasing XAF1 expression. The levels of XAF1 expression were determined in both the renal tissues of Thy- $1 \mathrm{~N}$ rats (in vivo) and cultured rat GMCs after sublytic C5b-9 stimulation (in vitro). The time course study showed that the expression of XAF1 protein both in vivo and in vitro increased at $2 \mathrm{~h}$, peaked at $6 \mathrm{~h}$ and then reduced at $12 \mathrm{~h}$ (Figures $1 \mathrm{a}$ and $\mathrm{b}$ ). Thus, we examined the expression of XAF1 both in vivo and in vitro at $6 \mathrm{~h}$ time point in the following studies. An immunofluorescent study showed that XAF1 expression was colocalized with C5b-9 deposits in the glomeruli of rats at $6 \mathrm{~h}$ after Thy- $1 \mathrm{~N}$ induction (Figure 1c), indicating that a portion of C5b-9-bound cells expressed XAF1 instead of undergoing lysis. Our study in vitro also demonstrated that a portion of C5b-9-bound GMCs were TUNEL (terminal deoxynucleotidyl transferase-mediated dUTP nick end-labeling) positive at $6 \mathrm{~h}$ after sublytic C5b-9 stimulation, indicating these cells were undergoing apoptosis (Supplementary Figure S1). To further confirm that the above-mentioned effects were in fact because of sublytic C5b-9 complexes, control cells were treated with different media containing $5 \%$ Thy-1 antibody (Thy-1 Ab), 5\% Thy- $1 \mathrm{Ab}+4 \%$ heat inactive serum (HIS), 5\% Thy-1 Ab + 4\% complement C6-deficient serum (C6DS), 5\% Thy-1 Ab + 4\% C6DS + C6 (0.5 or 2 mg/l) and $5 \%$ Thy $-1 \mathrm{Ab}+4 \% \mathrm{C} 6 \mathrm{DS}+\mathrm{PBS}$, respectively. The results showed that XAF1 expression was markedly upregulated only in the GMCs induced by sublytic C5b-9 (Figure 1d). Meanwhile, adding C6 back to C6DS could recover its ability to induce XAF1 expression and GMC apoptosis (Supplementary Figure S2). These data confirm that XAF1 expression and GMC apoptosis are induced by sublytic C5b-9 complexes.

To explore the role of $X A F 1$ gene in $\mathrm{GMC}$ apoptosis in response to sublytic C5b-9, the cultured GMCs were divided into the following six groups: (1) modified Eagle's medium (MEM), (2) sublytic C5b-9, (3) XAF1 short hairpin (sh)RNA (shXAF1) + sublytic C5b-9, (4) control shRNA (shCTR) + sublytic C5b-9, (5) pEGFP-N1/XAF1 and (6) pEGFP-N1 vector. The studies showed that XAF1 overexpression by using the plasmids of pEGFP-N1/XAF1 induced GMC apoptosis, whereas shXAF1 treatment reduced GMC apoptosis induced by sublytic C5b-9 stimulation (Figures $1 \mathrm{e}-\mathrm{g}$ ) but had no effect on the apoptosis of normal GMC without sublytic C5b-9 stimulation (Supplementary Figure S3). Moreover, XAF1 overexpression enhanced sublytic C5b-9-induced GMC apoptosis (Supplementary Figure S4). These findings suggest that $X A F 1$ gene expression might contribute to sublytic C5b-9-induced GMC apoptosis. In order to confirm that involvement of XAF1 in regulating GMC apoptosis is related to other stimulation, hydrogen peroxide $\left(\mathrm{H}_{2} \mathrm{O}_{2}\right)$ was used to induce GMC apoptosis, and the role of $\mathrm{XAF} 1$ was explored. The results showed that the expression of XAF1 was also involved in $\mathrm{H}_{2} \mathrm{O}_{2}$-induced $\mathrm{GMC}$ apoptosis at least in part (Supplementary Figure S5).

Sublytic C5b-9 induces GMC apoptosis via IRF-1regulated XAF1 expression. To determine whether IRF-1 activation of XAF1 is involved in sublytic C5b-9-induced GMC apoptosis, the cultured GMCs were divided into six groups of (1) MEM, (2) sublytic C5b-9, (3) IRF-1 shRNA (shIRF-1) + sublytic C5b-9, (4) control shRNA (shCTR) + sublytic C5b-9, (5) pcDNA3.1/IRF-1 and (6) pcDNA3.1 vector. As presented in Figure 2, overexpression of IRF-1 by using pcDNA3.1/IRF-1 markedly increased XAF1 expression (Figure 2a) and GMC apoptosis (Figures $2 \mathrm{~b}$ and $\mathrm{c}$ ), whereas treatment of GMCs with shIRF-1 inhibited XAF1 expression (Figure 2a) and GMC apoptosis (Figures 2b and c) stimulated by sublytic C5b-9. To further test whether sublytic C5b-9 can affect XAF1 gene transcription through IRF-1 expression, the GMCs were transfected with shIRF-1 or pcDNA3.1/IRF-1 together with pGL3-XAF1-full-length (pGL3-XAF1-FL) for $48 \mathrm{~h}$, and then the GMCs were treated with sublytic C5b-9 for another $6 \mathrm{~h}$. The luciferase analysis showed that the overexpression of IRF-1 significantly enhanced XAF1 promotor activity in the GMCs, whereas silence of IRF-1 gene obviously suppressed XAF1 transcriptional activity in the GMCs exposed to sublytic C5b-9 (Figure 3a). These data implicate that sublytic C5b-9 could upregulate $X A F 1$ gene expression and further increase GMC apoptosis via enhancing IRF-1 expression. 
a

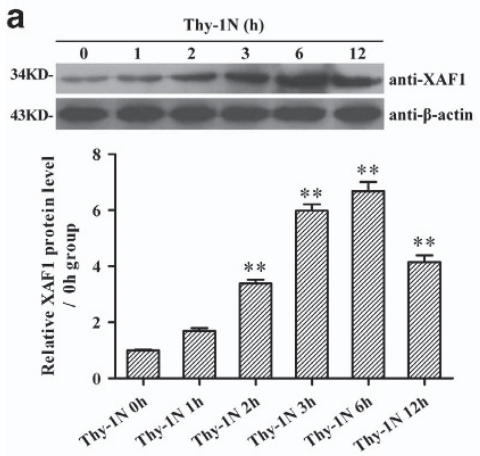

$43 \mathrm{KI}-$

b

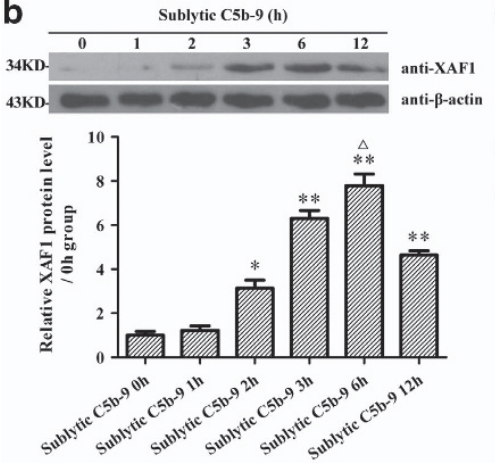

d
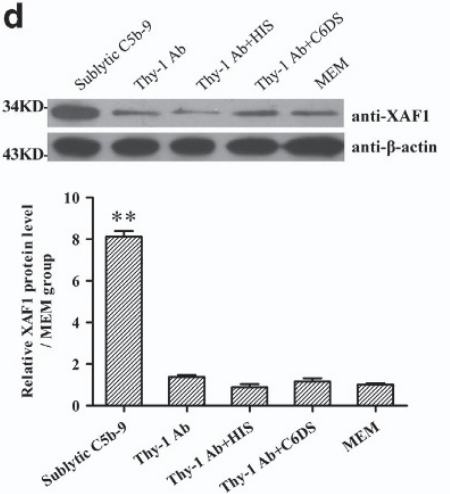

e
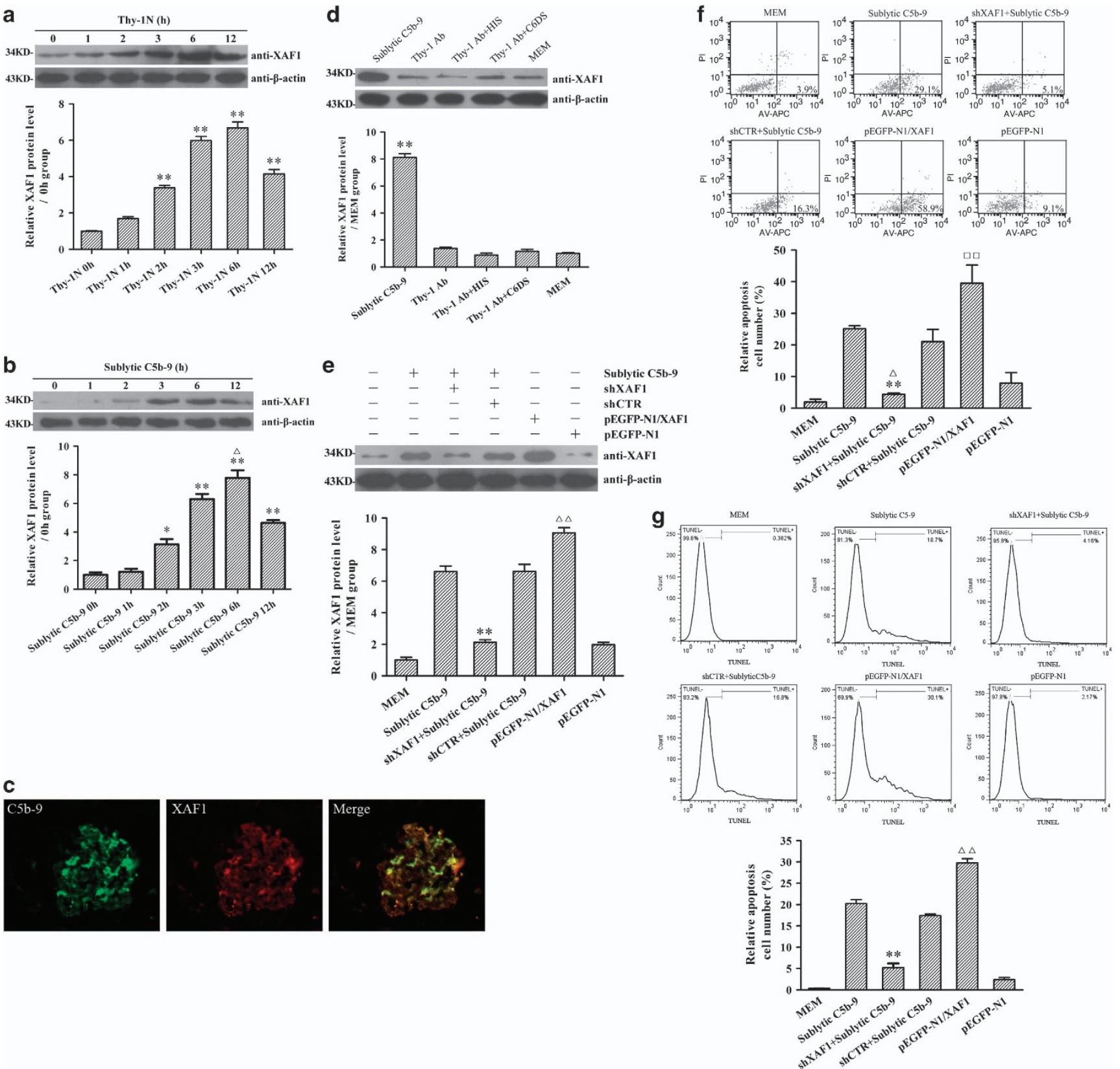

Figure 1 The expression and role of XAF1 in both renal tissues of rats with Thy-1N and GMCs exposed to sublytic C5b-9. (a) The level of XAF1 in the renal tissues of Thy- $1 \mathrm{~N}$ rats at varying time points was detected by IB analysis $\left(n=6\right.$ in each time point). ${ }^{* *} P<0.01$ versus $0 \mathrm{~h}$ group (nontreated). (b) Rat GMCs were treated with sublytic C5b-9 (5\% Thy-1 Ab + 4\% NHS) for the different time points, and then the level of XAF1 in the GMCs was determined by IB assay ( $n=3$ in each time point). ${ }^{*} P<0.05$, ${ }^{* *} P<0.01$ versus 0 h group (nontreated); ${ }^{\Delta} P<0.05$ versus other time points. (c) C5b-9 complex deposits (green) and XAF1 expression (red) in the glomeruli of Thy- $1 \mathrm{~N}$ rats at $6 \mathrm{~h}$ were detected by immunofluorescence staining. (d) GMCs were cultured for $6 \mathrm{~h}$ in dissimilar medium, namely, sublytic C5b-9 (5\% Thy $-1 \mathrm{Ab}+4 \% \mathrm{NHS}), 5 \%$ Thy- $1 \mathrm{Ab}, 5 \%$ Thy $-1 \mathrm{Ab}+4 \% \mathrm{HIS}, 5 \%$ Thy- $1 \mathrm{Ab}+4 \%$ C6DS and MEM. XAF1 expression was then determined by IB analysis ( $n=3$ in every group). ${ }^{* *} P<0.01$ versus Thy $-1 \mathrm{Ab}$, Thy- 1 $A b+$ HIS, Thy-1 Ab + C6DS and MEM group. (e-g) GMCs were divided into the following six groups: (1) MEM, (2) sublytic C5b-9, (3) shXAF1 + sublytic C5b-9, (4) shCTR + sublytic C5b-9, (5) pEGFP-N1/XAF1 and (6) pEGFP-N1. (e) IB assay was used to detect XAF1 expression in different groups of GMC at $6 \mathrm{~h}$ after sublytic C5b-9 stimulation ( $n=3$ in each group). ${ }^{* *} P<0.01$ versus sublytic C5b-9 group and shCTR + sublytic C5b-9 group; ${ }^{\Delta \Delta} P<0.01$ versus MEM group and $p E G F P-N 1$ group. (f) Both annexin V-APC and propidium iodide were used to label the cells in the above-mentioned groups at $6 \mathrm{~h}$ after sublytic C5b-9 stimulation. Flow cytometry analysis was performed to detect the numbers of annexin V-positive GMC ( $n=3$ in each group). ${ }^{* *} P<0.01$ versus sublytic C 5 b-9 group; ${ }^{\wedge} P<0.05$ versus shCTR + sublytic C5b-9 group; $\square \square P<0.01$ versus MEM group and pEGFP-N1 group. (g) TUNEL staining (TMR-labeled) was used to label the apoptotic cells in the above-mentioned groups at $6 \mathrm{~h}$ after sublytic $\mathrm{C} 5 \mathrm{~b}-9$ stimulation. Flow cytometry analysis was performed to detect the numbers of TUNEL-positive GMCs ( $n=3$ in each group). ${ }^{* *} P<0.01$ versus sublytic C5b-9 group and shCTR + sublytic C5b-9 group; ${ }^{\Delta \Delta} P<0.01$ versus MEM group and pEGFP-N1 group. The data are from one experiment, representative of three independent experiments. Results were represented as means \pm S.E. Representative photographs were manifested 


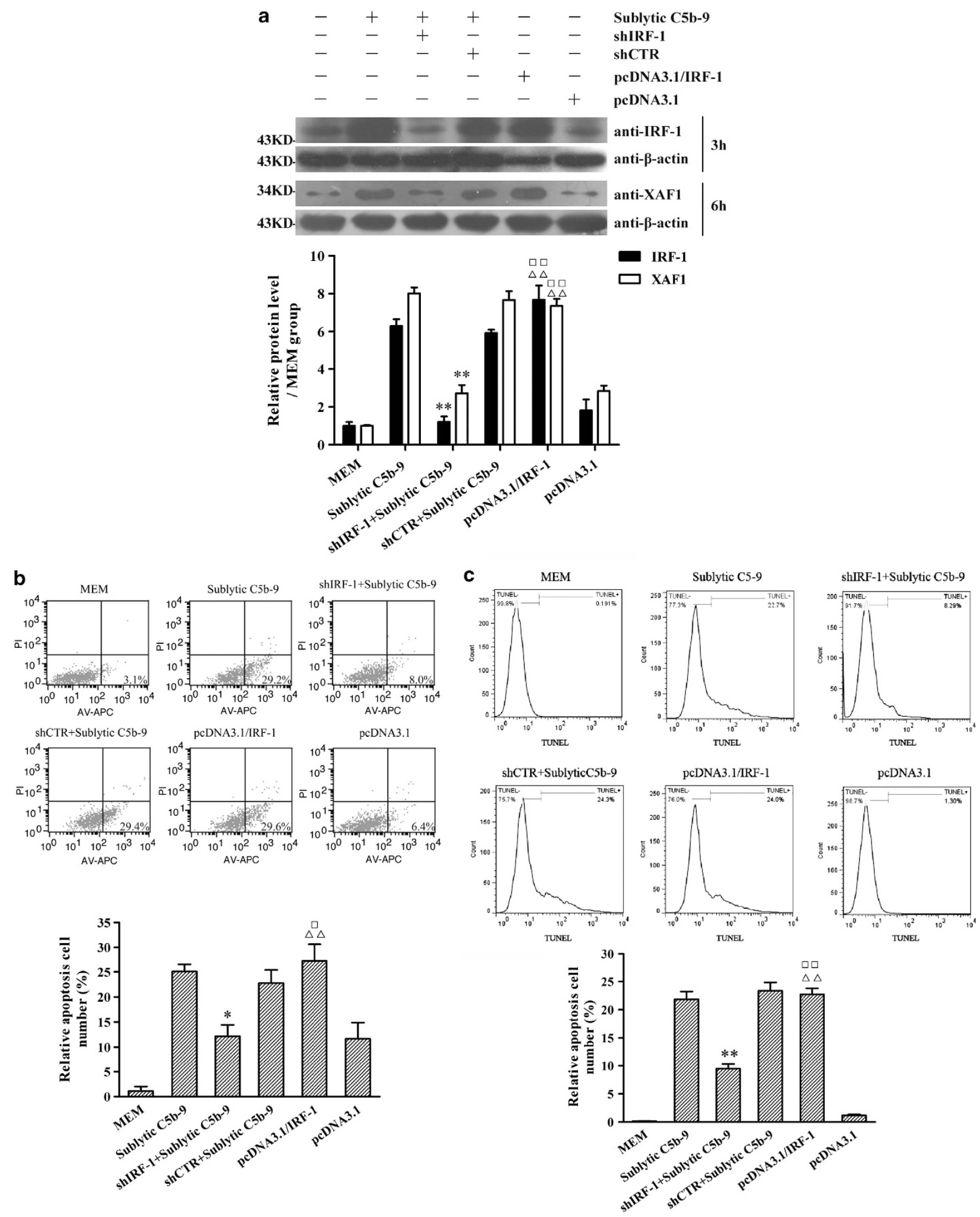

Figure 2 The roles of IRF-1 protein expression in XAF1 gene expression and GMC apoptosis upon sublytic C5b-9 attack. GMCs were divided into six groups of (1) MEM, (2) sublytic C5b-9, (3) shIRF-1 + sublytic C5b-9, (4) shCTR + sublytic C5b-9, (5) pcDNA3.1/IRF-1 and (6) pcDNA3.1. (a) The expression of IRF-1 and XAF1 in GMCs at 3 and $6 \mathrm{~h}$, respectively, after sublytic C5b-9 stimulation was detected by using IB assay. (b) Both annexin V-APC and propidium iodide were used to label the cells at $6 \mathrm{~h}$ after sublytic C5b-9 stimulation. The number of annexin V-positive GMCs was found by using flow cytometry analysis. (c) TUNEL staining (TMR-labeled) was used to label the apoptotic cells in different groups at $6 \mathrm{~h}$ after sublytic C5b-9 stimulation. Flow cytometry analysis was performed to detect the numbers of TUNEL-positive GMCs ( $n=3$ in each group). The data are from one experiment, representative of three independent experiments. Results were represented as means \pm S.E. ( $n=3$ in each group). Representative photographs were manifested. ${ }^{*} P<0.05,{ }^{\star \star} P<0.01$ versus sublytic $C 5 b-9$ group and shCTR + sublytic $C 5$ b-9 group; ${ }^{\Delta \Delta} P<0.01$ versus MEM group; ${ }^{\square} P<0.05,{ }^{\square} P<0.01$ versus pcDNA3.1 group 
a
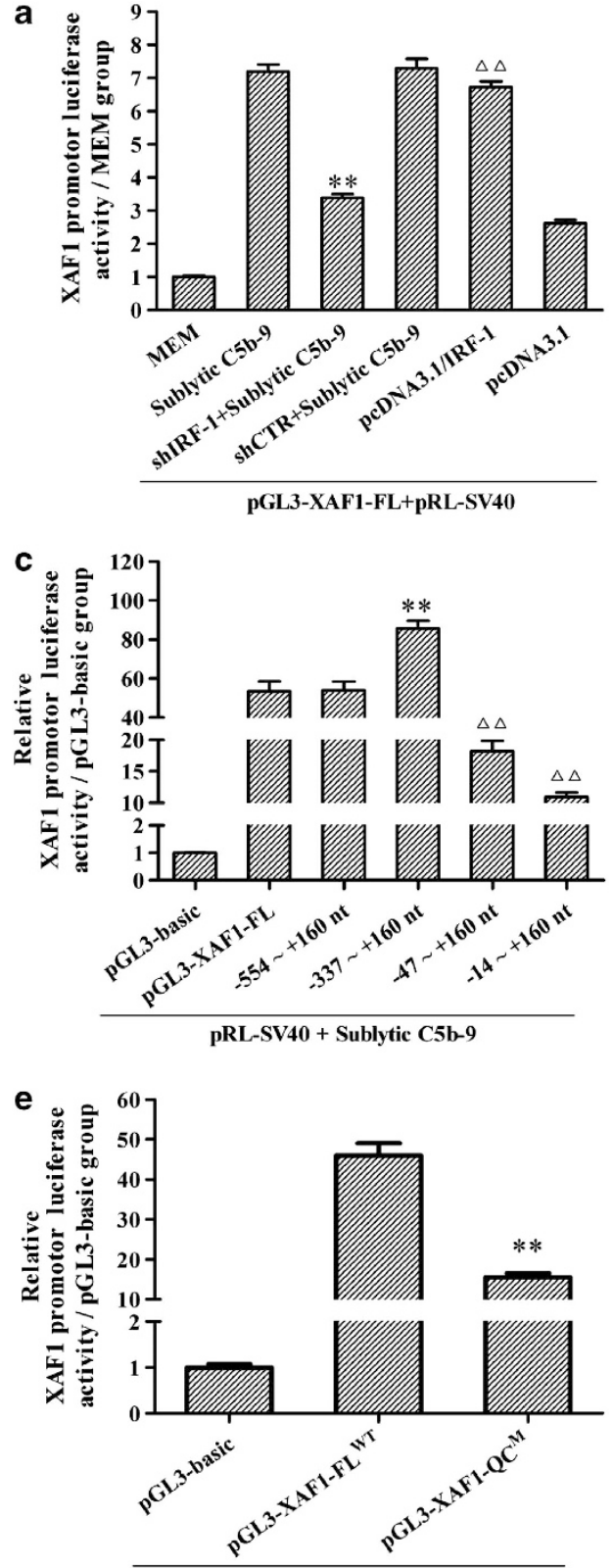

pRL-SV40 + pcDNA3.1/IRF-1

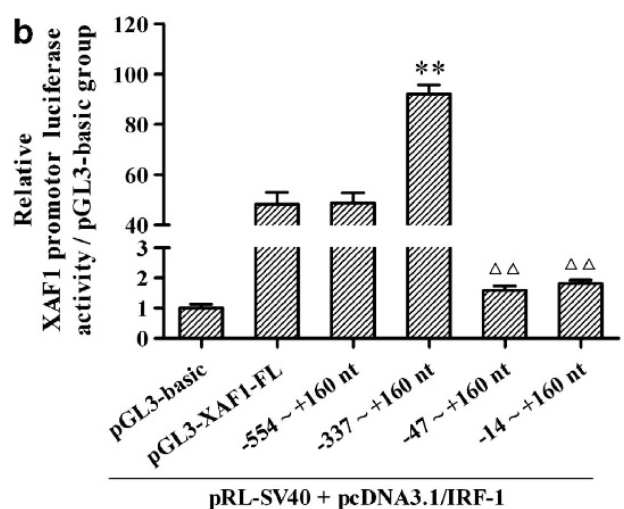

d

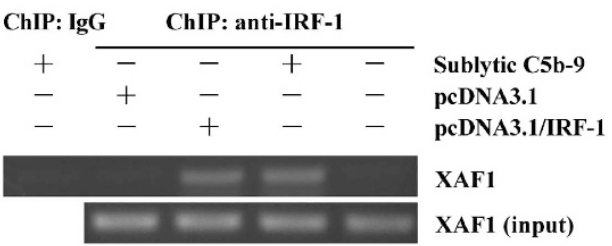

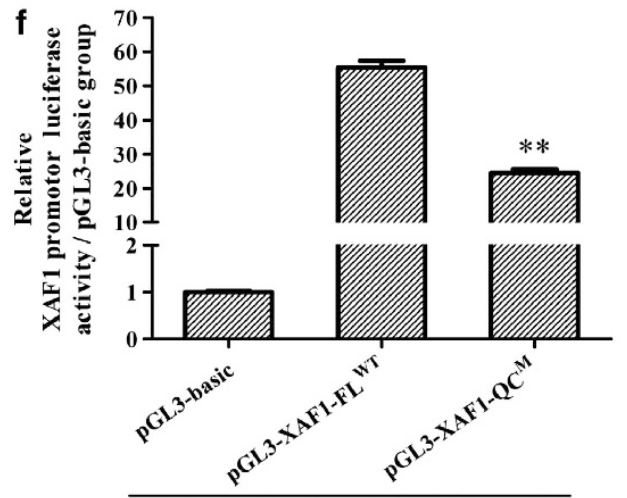

pRL-SV40 + Sublytic C5b-9

Figure 3 The effects of IRF-1 expression on the activity of XAF1 gene promoter in the GMCs exposed to sublytic C5b-9. (a) XAF1 promotor activity in different groups of GMC at $6 \mathrm{~h}$ after sublytic $C 5 \mathrm{~b}-9$ stimulation was determined by luciferase analysis. ${ }^{* *} P<0.01$ versus sublytic $C 5 \mathrm{~b}-9$ group and shCTR + sublytic $C 5 \mathrm{~b}-9$ group; ${ }^{\Delta \Delta} P<0.01$ versus MEM group and pcDNA3.1 group. (b and $\mathbf{c}$ ) The GMCs were transfected with the luciferase reporter constructs containing the $1.65-\mathrm{kb}$ XAF1 promoter ( -1496 to $+160 \mathrm{nt}$ ) or different promoter deletion fragments $(-554$ to $+160,-337$ to $+160,-47$ to +160 and -14 to $+160 \mathrm{nt}$ ) accompanied with pcDNA3.1/IRF-1 for $48 \mathrm{~h}$ (b) or sublytic C5b-9 stimulation for $6 \mathrm{~h}$ (c), and the activity of XAF1 promoter was determined subsequently. ${ }^{* \star} P<0.01$ versus pGL3-XAF1-FL group; ${ }^{{ }^{\Delta}} P<0.01$ versus pGL3XAF1-FL group, pGL3-XAF1 ( -554 to $+160 \mathrm{nt}$ ) group and pGL3-XAF1 $(-337$ to $+160 \mathrm{nt})$ group. (d) ChIP assay was performed using the anti-IRF-1 Ab and preimmune IgG respectively, and immunoprecipitated DNA was amplified using one pair of primers for the proximal promoter region ( -169 to $-19 \mathrm{nt}$ ) of the XAF1 gene subsequently. Representative photographs were manifested. (e and $\mathbf{f}$ ) Luciferase analysis was performed to determine the effects of XAF1 promotor mutation $(-65$ to $-53 \mathrm{nt}$ region) on the transcription activity of pGL3-XAF1 induced by both IRF-1 overexpression ( $48 \mathrm{~h})$ and sublytic $\mathrm{C} 5 \mathrm{~b}-9$ stimulation $(6 \mathrm{~h}) .{ }^{* \star} \mathrm{P}<0.01$ versus pGL3-XAF1-FL ${ }^{\mathrm{WT}}$ group. The data are from one experiment, representative of three independent experiments. Results were represented as means \pm S.E. ( $n=3$ in each group)

Identification of IRF-1 binding to rat XAF1 promoter element. To identify the effective promoter regions required for $X A F 1$ gene transcription in response to IRF-1 expression and sublytic C5b-9 stimulation, the GMCs were transfected with the luciferase reporter constructs of pGL3-XAF1-FL or different promoter deletion fragments $(-554$ to +160 ,
-337 to $+160,-47$ to +160 and -14 to $+160 \mathrm{nt})$ accompanied with pcDNA3.1/IRF-1 for $48 \mathrm{~h}$ or sublytic C5b-9 stimulation for $6 \mathrm{~h}$, and the activity of XAF1 promoter was determined subsequently. As presented in Figures $3 \mathrm{~b}$ and $\mathrm{c}$, the GMCs transfected with XAF1 promoter $(-47$ to $+160 \mathrm{nt})$ showed markedly decreased luciferase activity 
relative to the other groups, suggesting that the region of rat XAF promoter $(-337$ to $-47 \mathrm{nt})$ might contain an IRF-1binding element.

TFsearch software (http://www.cbrc.jp/research/db/ TFSEARCH.html) predicted that an IRF-E ( -65 to $-53 \mathrm{nt})$ is located within the region of rat $X A F 1$ gene promoter ( -337 to $-47 \mathrm{nt})$. Chromatin immunoprecipitation (ChIP) assay revealed that both sublytic C5b-9-induced IRF-1 and overexpressed IRF-1 by using pEGFP-N1/XAF1 could bind to this element (Figure $3 d$ ). To further confirm that sublytic C5b9-induced XAF1 gene transcription could be mediated by the IRF-E, we mutated the IRF-E (pGL3-XAF1 ${ }^{\mathrm{M}}$ ). As shown in Figures $3 e$ and $f,-65$ to -53 nt region mutation abrogated the activity of $X A F 1$ gene promoter induced by both IRF-1 overexpression and sublytic $\mathrm{C} 5 \mathrm{~b}-9$ stimulation. To further identify the interaction between IRF-1 protein and XAF1 gene promoter, the plasmids of $\mathrm{pGL3}-\mathrm{XAF} 1-\mathrm{FL}{ }^{\mathrm{WT}}$ and $\mathrm{pGL3}-\mathrm{XAF} 1^{\mathrm{M}}$ were transfected into 293T cells, respectively, accompanied with pcDNA3.1/IRF-1. Luciferase reporter assay displayed that exogenous IRF-1 markedly promoted the transcription of wildtype XAF1 gene but not mutated XAF1 gene in 293T cells (Supplementary Figure S6). Collectively, the findings indicate that upregulation of XAF1 promoter activity in sublytic $\mathrm{C} 5 \mathrm{~b}-9$ induced GMCs depends on the increase of IRF-1 binding to the IRF-E ( -65 to $-53 \mathrm{nt}$ ) within XAF1 promoter.

\section{The p300-mediated IRF-1 acetylation is required for sublytic C5b-9-induced XAF1 expression and GMC} apoptosis. IRF-1 acetylation emerged in a time-dependent manner, with the maximum level at $3 \mathrm{~h}$ after exposure to sublytic C5b-9 (Figures 4a and b). Reportedly, CREB-binding protein (CBP), p300 and P300/CBP-associated factor (PCAF) are important transcription coactivators that could interact with multiple transcription factors as acetyl transferases. $^{38-40}$ Thus, the expression levels of CBP, p300 and PCAF were further detected, and we found that sublytic C5b9-induced GMCs exhibited a time-dependent increase of CBP, p300 and PCAF expression that reached the maximum at $3 \mathrm{~h}$ (Figure $4 \mathrm{c}$ ), indicating that the expression of CBP, p300 and PCAF perhaps correlated with IRF-1 acetylation. Co-immunoprecipitation (co-IP) assay further revealed that only p300 markedly interacted with IRF-1 at the protein level in the GMCs induced by sublytic C5b-9 (Figure 4d and Supplementary Figure S7). Meanwhile, adding C6 back to C6DS could recover its ability to induce the combination of p300 and IRF-1, and IRF-1 acetylation (Supplementary Figure S8). Therefore, p300 was chosen for subsequent studies, and the results showed that silencing p300 gene not only obviously inhibited IRF-1 acetylation, but also markedly reduced the binding of IRF-1 to XAF-1 promotor and XAF1 expression as well as GMC apoptosis because of sublytic C5b-9 stimulation (Figures $4 \mathrm{e}-\mathrm{h}$ ).

Further studies were designed to determine whether p300 can bind to $X A F 1$ gene promotor ( -65 to $-53 \mathrm{nt}$ ) together with IRF-1 as a complex, and p300 itself can directly bind to $X A F 1$ protein and induce the acetylation of XAF1. In Figure 4i, re-ChIP analysis showed that the complex of p300 and IRF-1 bound to the $X A F 1$ gene promotor through IRF-1 binding to its element ( -65 to $-53 \mathrm{nt})$. Meanwhile, co-IP assay showed that $\mathrm{p} 300$ did not bind to XAF1 at the protein level, and XAF1 could not be acetylated in sublytic C5b-9-induced GMCs (Supplementary Figure S9). To observe whether IRF-1 acetylation may crosstalk with other post-translational modifications such as ubiquitination, IRF-1 ubiquitination was further detected, and the data showed that shp300 had no effect on IRF-1 ubiquitination (Supplementary Figure S10). Together, these data support the idea that sublytic C5b-9 complexes could induce GMC apoptosis through p300regulated IRF-1 acetylation that could enhance XAF1 gene transcription.

Identification of IRF-1 acetylation sites. To find the IRF-1 acetylation sites, N-terminal Lys-29, Lys-39, Lys-50, Lys-66, Lys-70, Lys-75 and Lys-78 of IRF-1 within the DNA-binding domain were mutated respectively, and the IRF-1 acetylation, XAF1 expression and GMC apoptosis in response to IRF-1 overexpression were detected subsequently. The data showed that site mutation of Lys-39 and Lys-78 not only reduced IRF-1 acetylation (Figures $5 \mathrm{a}$ and b) and IRF-1 binding to $X A F 1$ gene promotor (Figure $5 \mathrm{c}$ ), but also suppressed XAF1 expression (Figures $5 b$ and $d$ ) and subsequent GMC apoptosis (Figures $5 e$ and f).

\footnotetext{
Figure 4 The effects of p300 on IRF-1 expression, XAF1 gene activation and GMC apoptosis exposed to sublytic C5b-9. (a) Rat GMCs were treated with sublytic C5b-9 for the fixed time, and then the Abs against IRF-1 and preimmune IgG were used to perform IP respectively. Subsequently, the content of IRF-1 and acetylated lysine in the complexes was determined by IB assay. Meanwhile, the expression of $\beta$-actin and IRF-1 in WCE was detected by IB assay. (b) GMCs were cultured for $3 \mathrm{~h}$ in dissimilar medium, namely, sublytic C5b-9 (5\% Thy-1 Ab + 4\% NHS), 5\% Thy-1 Ab, 5\% Thy-1 Ab + 4\% HIS, 5\% Thy-1 Ab + 4\% C6DS and MEM. The Abs against IRF-1 and preimmune IgG were used to perform IP respectively and then the content of IRF-1 and acetylated lysine in the complexes was measured by IB assay. Meanwhile, the expression of $\beta$-actin in WCE was detected by IB assay. (c) Rat GMCs were treated with sublytic C5b-9 for the indicated times, and then the levels of CBP, p300 and PCAF were determined by IB. (d) GMCs were cultured for $3 \mathrm{~h}$ in the above-mentioned five groups. The Abs against IRF-1 and preimmune IgG were used to perform co-IP respectively, and then the content of CBP, p300 and PCAF in the complexes was examined by IB assay. Meanwhile, the expression of corresponding protein in WCE was detected by IB assay. (e) The Abs against IRF-1 and preimmune IgG were used to perform co-IP respectively, and then the content of IRF-1, p300 and acetylated lysine in the complexes was detected by IB assay at 3 and $6 \mathrm{~h}$, respectively, after sublytic C5b-9 stimulation. Meanwhile, the expression of corresponding protein in WCE was detected by IB assay. (f) ChIP assay was performed using the anti-IRF-1 Ab, and immunoprecipitated DNA was amplified using a pair of primers for the proximal promoter region ( -169 to $-19 \mathrm{nt}$ ) of the XAF1 gene subsequently. (g) Both annexin V-APC and propidium iodide were used to label the GMC in different groups at $6 \mathrm{~h}$ after sublytic C5b-9 stimulation. Flow cytometry analysis was performed to detect the numbers of annexin V-positive GMCs. (h) TUNEL staining (TMR-labeled) was used to label the cells in different groups at $6 \mathrm{~h}$ after sublytic C5b-9 stimulation. Flow cytometry analysis was performed to detect the numbers of TUNEL-positive GMCs. (i) A ChIP assay was performed by using the Ab against IRF-1 and preimmune IgG, respectively, and subsequently a re-ChIP assay was performed by using the Ab against p300 and preimmune IgG, respectively. Immunoprecipitated DNA was then amplified by using a pair of primers for the proximal promotor region ( -169 to $-19 \mathrm{nt})$ of the XAF1 gene. The results were normalized to input. The data are from one experiment, representative of three independent experiments or four independent experiments (c). Results were represented as means \pm S.E. ( $n=3$ in each group). Representative photographs were manifested. ${ }^{\star} P<0.05$, ${ }^{\star *} P<0.01$ versus 0 h group (nontreated); $\square \square P<0.01$ versus sublytic C5b-9 group and shCTR + sublytic C5b-9 group
} 
Knockdown of p300, IRF-1 and XAF1 genes suppresses GMC apoptosis and other pathologic changes as well as urinary protein of the rats with Thy-1N. First, the time course study showed that the expression of p300 and IRF-1 markedly increased at $3 \mathrm{~h}$ after Thy- $1 \mathrm{~N}$ induction simultaneously, and then gradually decreased (Figure 6a). Moreover, co-IP assay found marked IRF-1 acetylation and enhanced combination between p300 and IRF-1 protein in the renal tissues of Thy- $1 \mathrm{~N}$ rats at $3 \mathrm{~h}$ (Figure $6 \mathrm{~b}$ ), consistent with our above-mentioned results in vitro.
To further evaluate the roles of p300, IRF-1 and XAF1 expression in GMC apoptosis and secondary proliferation of Thy-1N rats, normal Sprague-Dawley (SD) rats were divided into the following six groups: (1) normal serum (NS), (2) Thy-1N, (3) Lv-shp300 + Thy-1N, (4) Lv-shIRF-1 + Thy-1N, (5) Lv-shXAF1 + Thy-1N and (6) Lv-shCTR + Thy-1N. The protein levels of $\mathrm{p} 300, \mathrm{IRF}-1$ and XAF1 in rat renal tissues were determined at 3 and $6 \mathrm{~h}$ respectively after nephritis induction. The data showed that pretreatments of rats with Lv-shp300, Lv-shIRF-1 and Lv-shXAF1 decreased the expression a
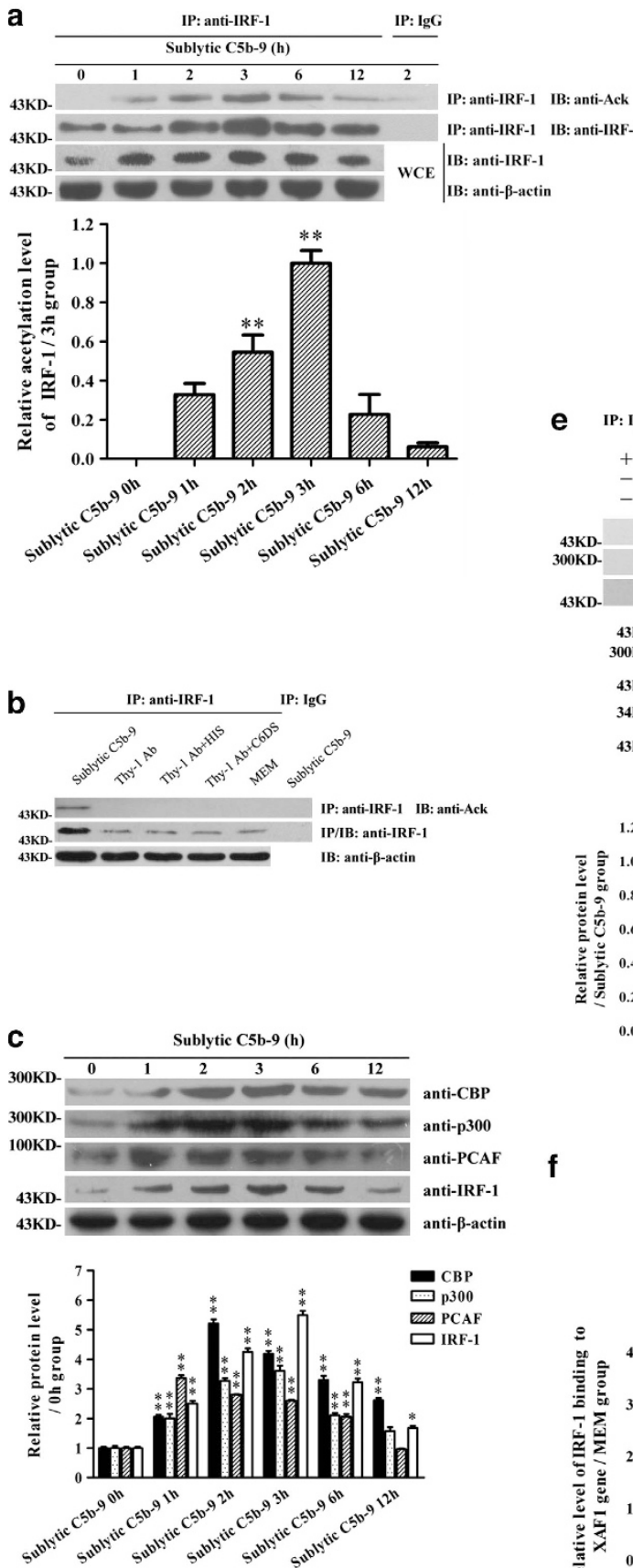

f d

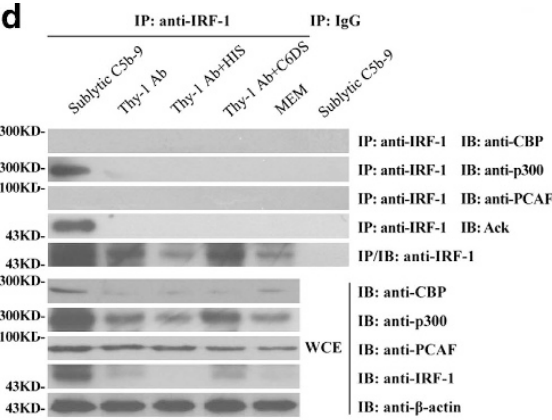

e IP: IgG

IP: anti-IRF-1
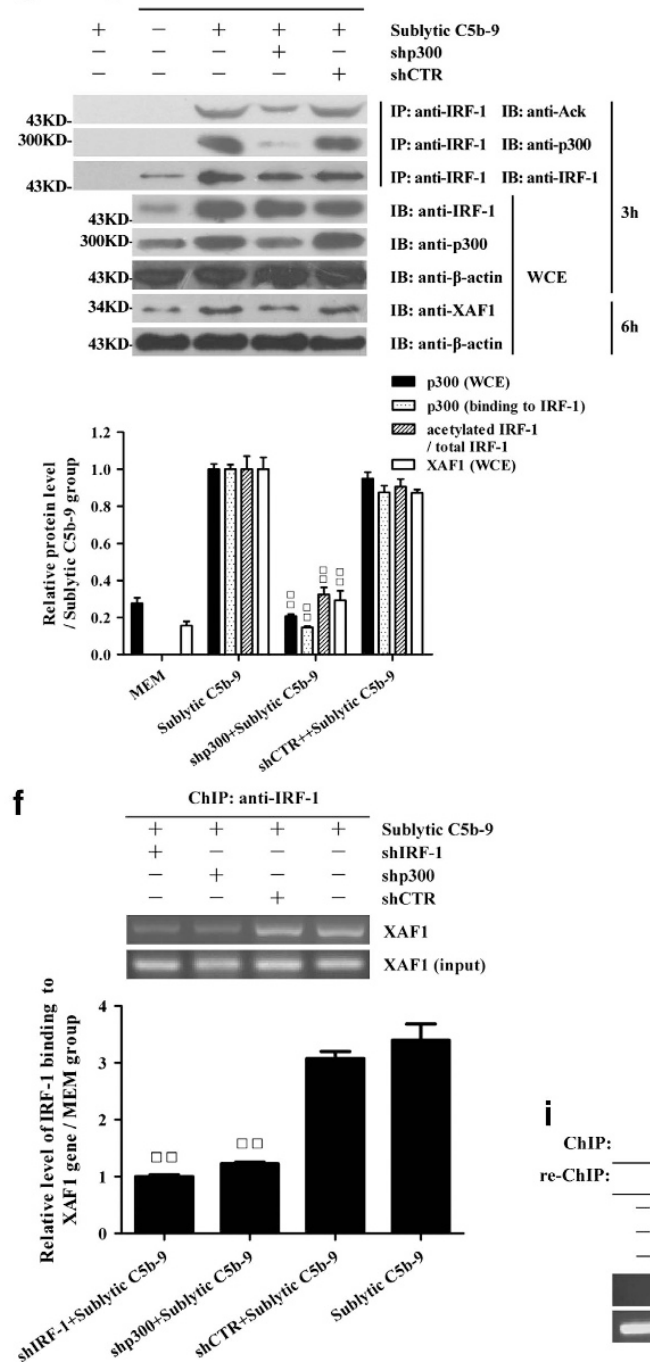

g
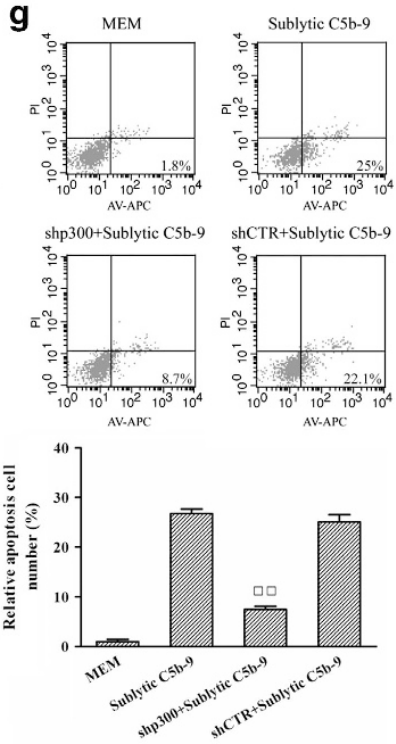

h
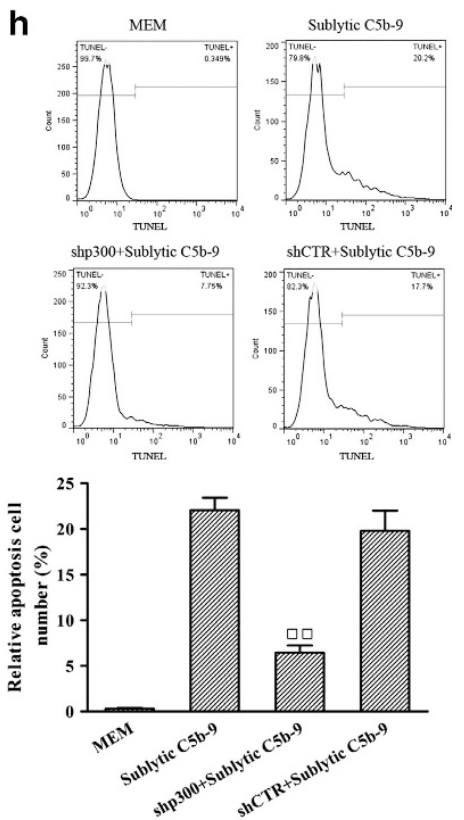

i

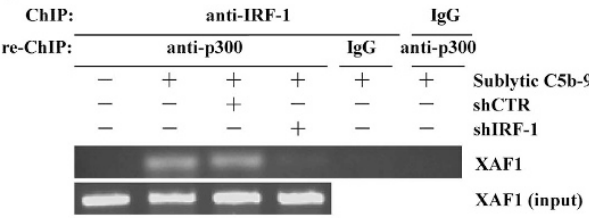


8
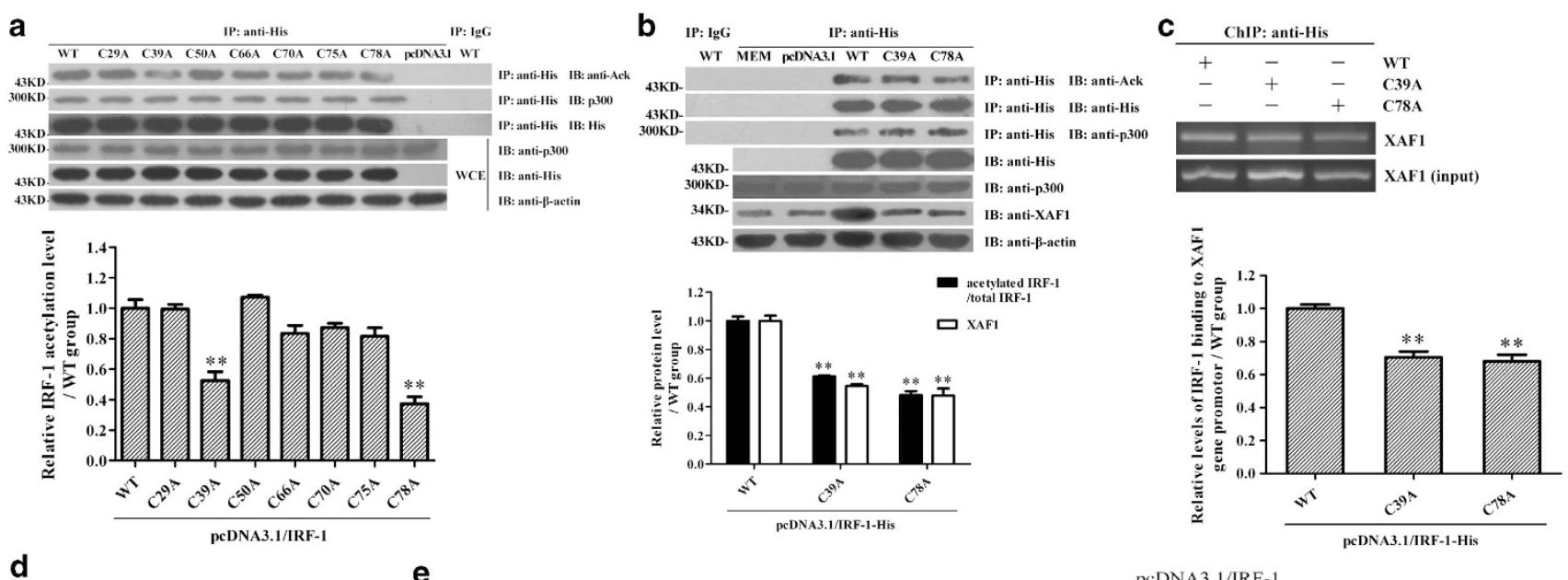

e
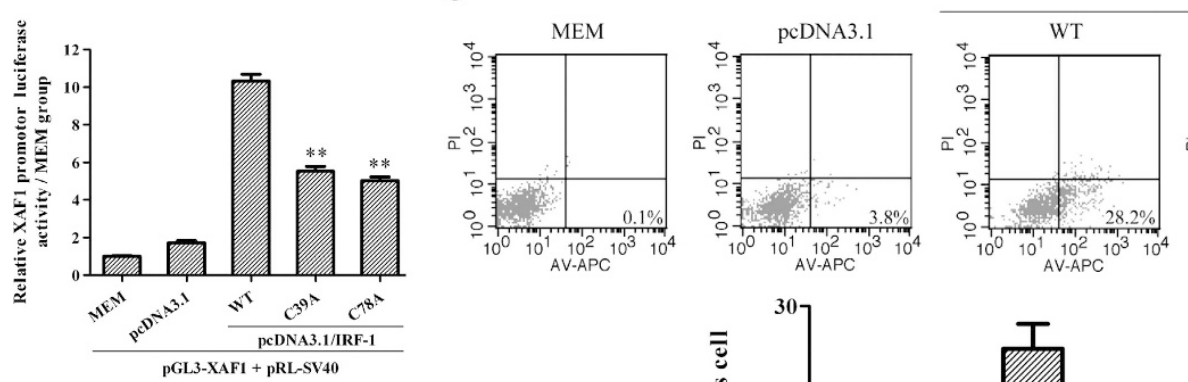

peDNA3.1/IRF-1
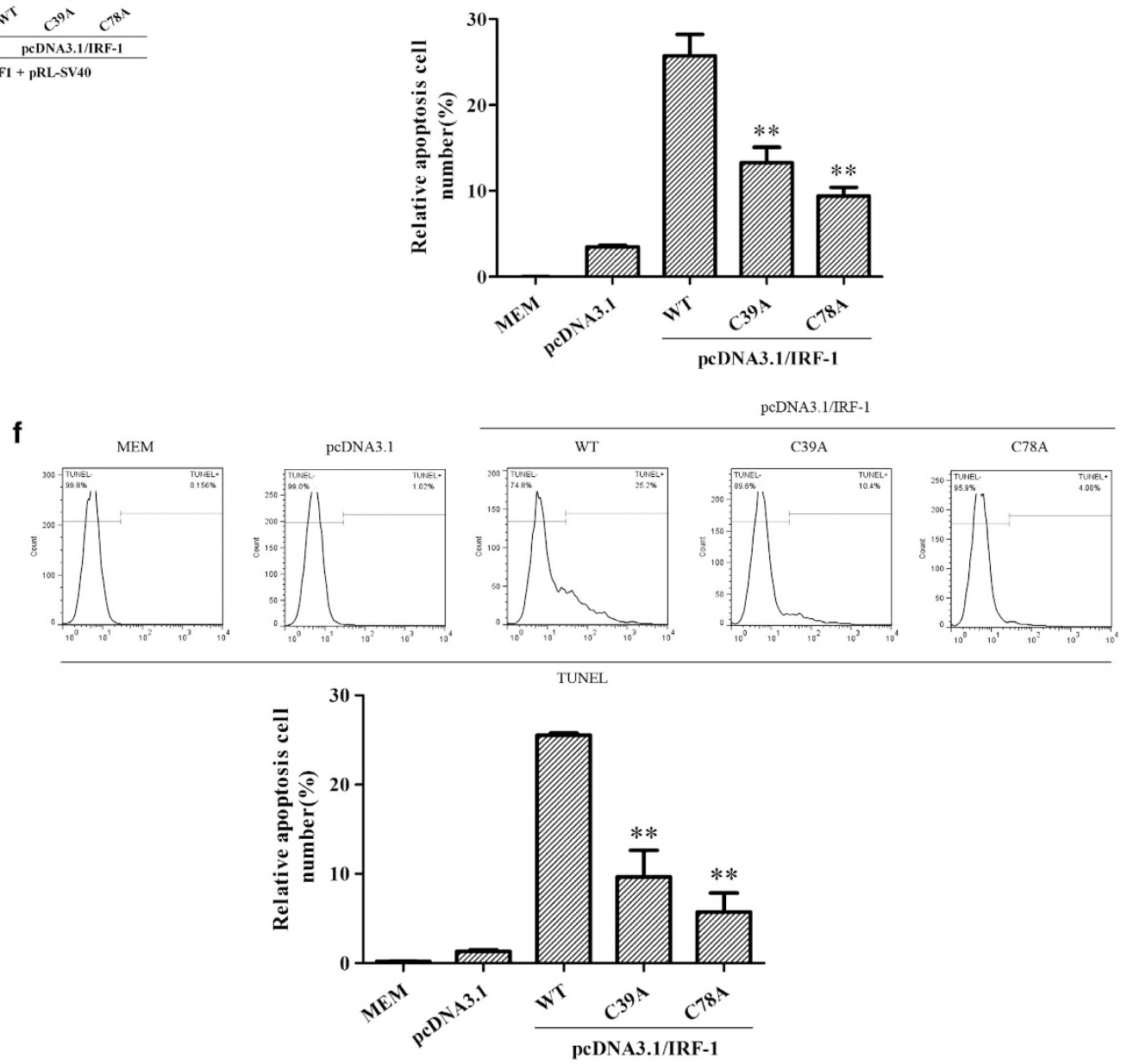
of p300, IRF-1 and XAF1 in the renal tissues, respectively. On the other hand, the Lv-shp300 pretreatment inhibited IRF-1 acetylation and XAF1 expression, but had no effect on IRF-1 expression. Lv-shIRF-1 pretreatment suppressed XAF1 expression, whereas it had no effect on p300 production, and the Lv-shXAF1 pretreatment also had no effect on p300 and
IRF-1 expression as well as IRF-1 acetylation (Figures $6 \mathrm{c}$ and d), consistent with our above-mentioned results in vitro.

Furthermore, TUNEL staining and electron microscopy (EM) showed that all pretreatments of GMC with Lv-shp300, Lv-shIRF-1 and Lv-shXAF1 decreased the number of glomerular TUNEL-positive cells, and the apoptotic changes

b
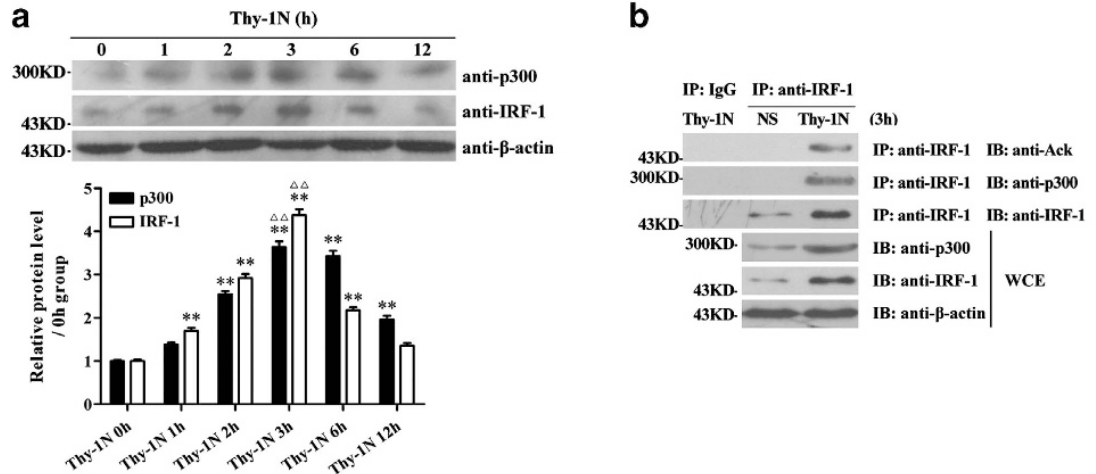

C
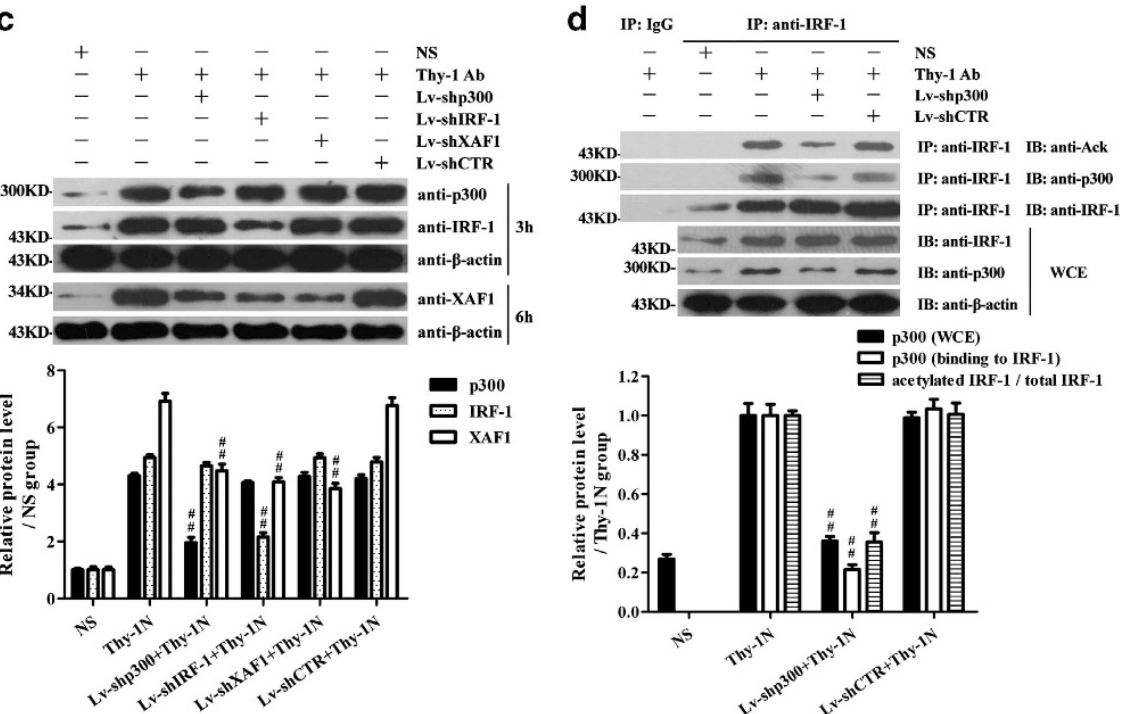

Figure 6 The expression and interaction of p300, IRF-1 and XAF1 in the renal tissues of Thy-1N rats. (a) The levels of $p 300$ and IRF-1 in the renal tissues of Thy-1N rats at varying time points were measured by IB analysis. (b) The level of IRF-1 acetylation as well as the combination of p300 and IRF-1 in the renal tissues of Thy-1N rats and NS control rats at $3 \mathrm{~h}$ were detected by co-IP assay. Lysates of renal tissues were used to perform co-IP by the anti-IRF-1 Ab to pull down endogenous IRF-1 (preimmune IgG as a control reaction), and then perform IB experiment for examining $\mathrm{p} 300$, IRF-1 and acetylated lysine expression. Meanwhile, the expression of $\beta$-actin, IRF-1 and p300 in WCE was detected by IB assay. (c) SD rats were divided into six groups, namely: (1) NS, (2) Thy-1N, (3) Lv-shp300 + Thy-1N, (4) Lv-shIRF-1 + Thy-1N, (5) Lv-shXAF1 + Thy-1N and (6) Lv-shCTR + Thy-1N. The expression of p300, IRF-1 and XAF1 in the renal tissues of Thy-1N rats at 3 and $6 \mathrm{~h}$, respectively, were examined by IB assay. (d) SD rats were divided into four groups, namely: (1) NS, (2) Thy-1N, (3) Lv-shp300 + Thy-1N and (4) Lv-shCTR + Thy-1N. Lysates of renal tissues at $3 \mathrm{~h}$ after nephritis induction were used to perform co-IP by the anti-IRF-1 Ab to pull down endogenous IRF-1 (preimmune IgG as a control reaction), and then perform IB experiment for p300, IRF-1 and acetylated lysine expression. Meanwhile, the expression of $\beta$-actin, IRF-1 and p300 in WCE was detected by IB assay. The data are from one experiment, representative of three independent experiments. Data are means \pm S.E. ( $n=6$ in each time point every group or $n=6$ in each group). The representative IB photographs are displayed. ${ }^{* *} P<0.01$ versus $0 \mathrm{~h}$ group (nontreated); ${ }^{\Delta \Delta} P<0.01$ versus other time points; ${ }^{\# \#} P<0.01$ versus Thy- $1 \mathrm{~N}$ group and Lv-shCTR + Thy- $1 \mathrm{~N}$ group

Figure 5 The identification of IRF-1 acetylation sites. To find the IRF-1 acetylation sites, N-terminal Lys-29, Lys-39, Lys-50, Lys-66, Lys-70, Lys-75 and Lys-78 of IRF-1 were mutated respectively, and the IRF-1 acetylation and XAF1 expression and GMC apoptosis were measured subsequently. (a and b) Rat GMCs were divided into different groups, and then the Abs against IRF-1 and preimmune IgG were used to perform IP respectively. Subsequently, the content of IRF-1 and acetylated lysine in the complexes was determined by IB assay. Meanwhile, the expression of corresponding protein in WCE was detected by IB assay. (c) The three plasmids of pcDNA3.1/IRF-1(WT)-His, pcDNA3.1/IRF-1(C39A)-His and pcDNA3.1/IRF-1(C78A)-His were transfected into GMCs for $48 \mathrm{~h}$ respectively. A ChIP assay was performed by using anti-His antibodies, and immunoprecipitated DNA was amplified by using a pair of primers for the proximal promotor region ( -169 to $-19 \mathrm{nt})$ of the XAF1 gene subsequently. The results were normalized to input. (d-f) Luciferase and flow cytometry analysis were performed to detect the effects of IRF-1 mutation (Lys-39 and Lys-78) on the XAF1 gene promotor activity (d) and GMC apoptosis (e, annexin V-APC and propidium iodide; $\mathbf{f}$, TUNEL staining, TMR-labeled). The data are from one experiment, representative of three independent experiments. Results were represented as means \pm S.E. ( $n=3$ in each group). Representative photographs were manifested. ${ }^{\star *} P<0.01$ versus wild-type IRF-1 overexpression group 

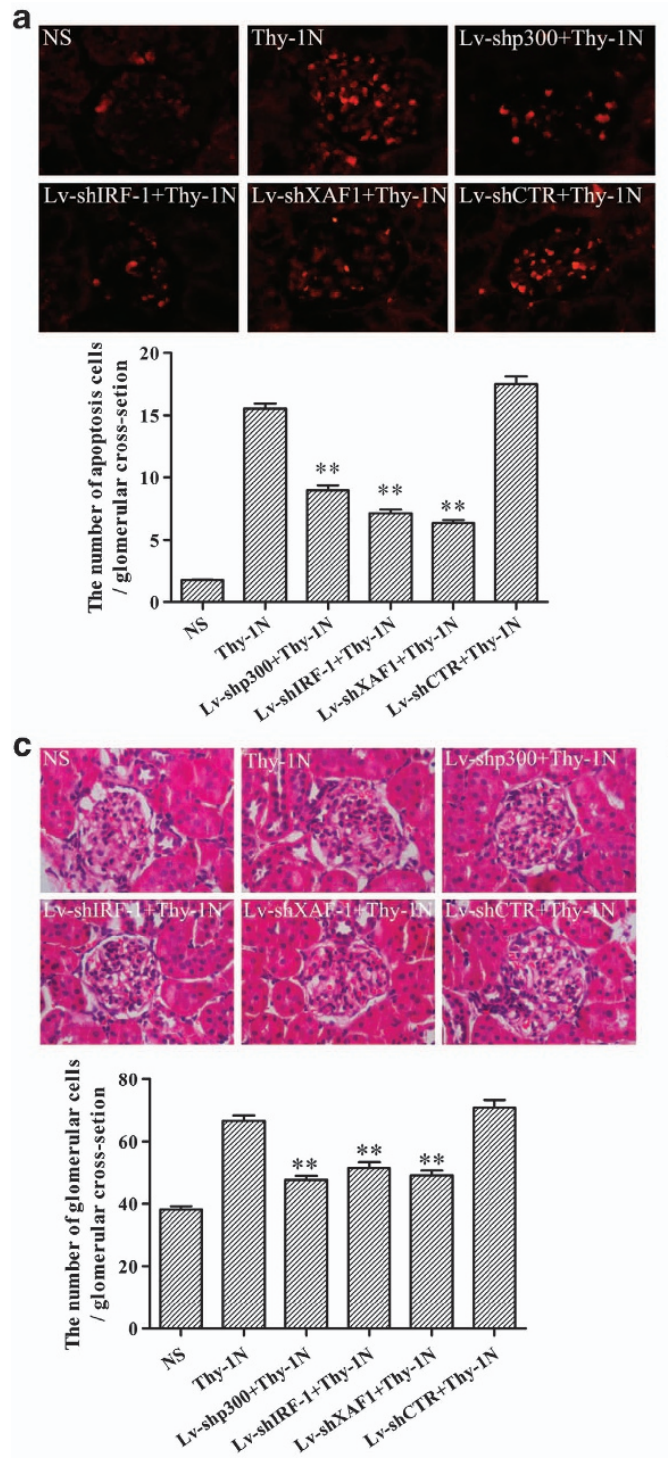

e

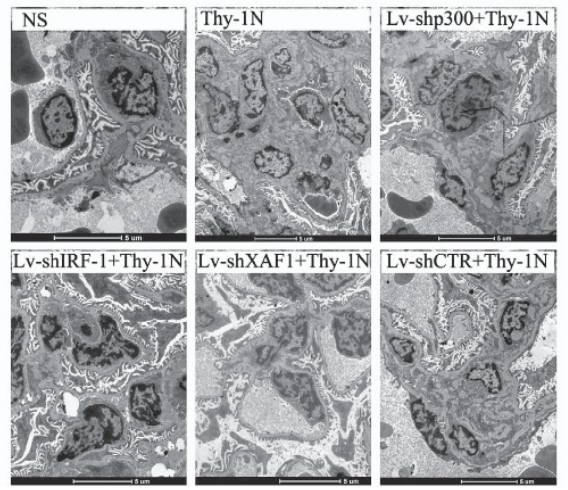

b
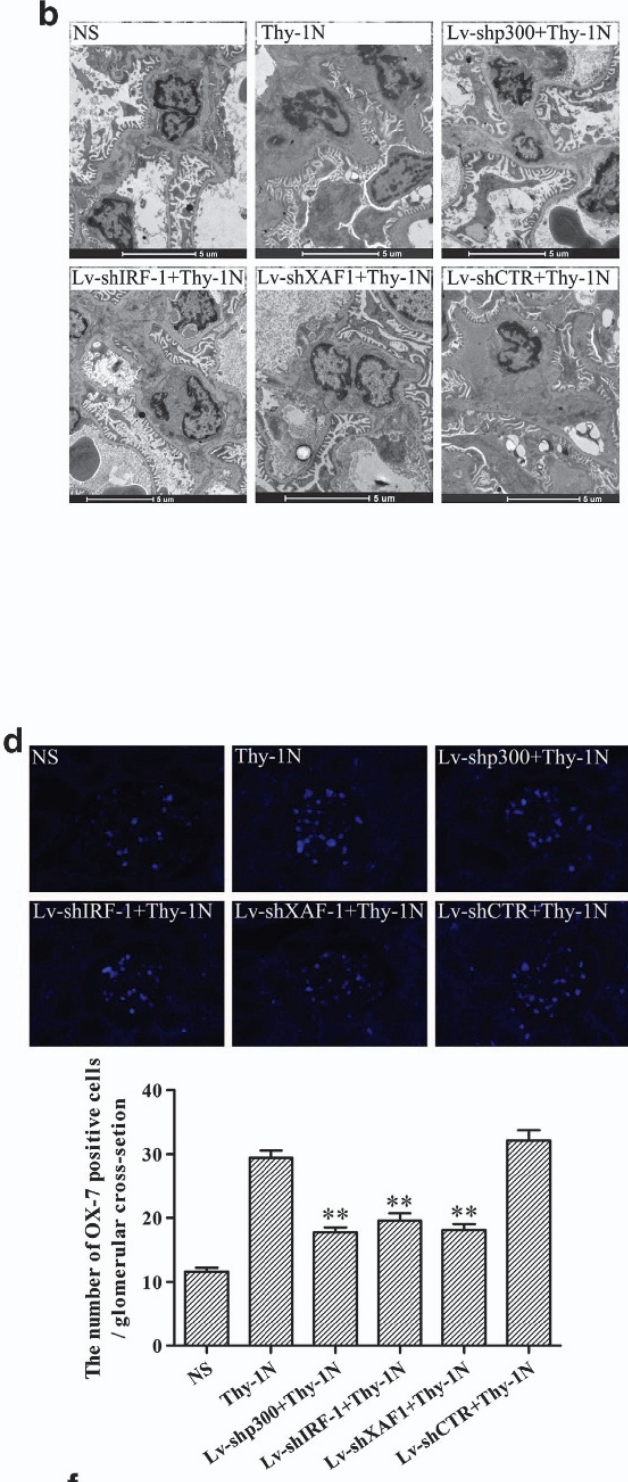

f

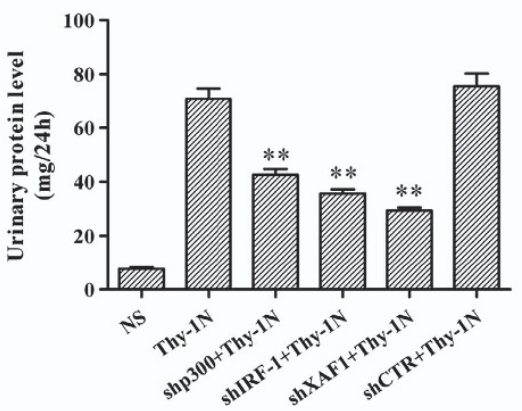

Figure 7 Effects of $p 300$, IRF-1 and XAF1 gene knockdown on the pathological changes in the renal tissues of Thy-1N rats. SD rats were divided into six groups, namely: (1) NS, (2) Thy-1N, (3) Lv-shp300 + Thy-1N, (4) Lv-shIRF-1 + Thy-1N, (5) Lv-shXAF1 + Thy-1N and (6) Lv-shCTR + Thy-1N. (a) TUNEL staining (frozen sections, original magnification: $\times 400$ ) was used to show the number of apoptosis cells in the six groups at $6 \mathrm{~h}$ after nephritis induction. (b) The ultrastructural changes at $6 \mathrm{~h}$ including the irregular aggregation of chromatin in the periphery of the nucleus and clear condensation of the nuclear chromatin were detected by EM. (c) The total number of glomerular cells in the 6 groups on day 7 after the nephritis induction was examined by HE staining (paraffin-embedded sections, original magnification: $\times 400$ ). (d) IF staining (frozen sections, original magnification: $\times 400$ ) for Thy-1 antigen (indicating GMC number) obtained similar results as HE staining. (e) EM was used to observe the ultrastructural changes including the number of GMC and the secretion of ECM in the six groups on day 7 after nephritis induction. (f) The amount of urinary protein ( $\mathrm{mg}$ per $24 \mathrm{~h}$ ) of rats in the six groups was detected on day 7. The data are from one experiment, representative of two independent experiments. Data are means $\pm S$.E. ( $n=6-8$ per group). Representative photographs were manifested. ${ }^{* *} P<0.01$ versus Thy- $1 \mathrm{~N}$ group and Lv-shCTR + Thy- $1 \mathrm{~N}$ group 
including irregular aggregation of chromatin at the periphery of the nucleus and condensation of the nuclear chromatin at $6 \mathrm{~h}$ after Thy-1N induction. EM analysis also demonstrated that GMCs were the main part of apoptotic cells (Figures 7a and b). In addition, HE staining, GMC staining, EM and urinary protein analysis showed that each of the pretreatment by using Lv-shp300, Lv-shIRF-1 and Lv-shXAF1 decreased the numbers of glomerular cells especially GMCs (Figures 7c-e) and urinary protein content ( $\mathrm{mg}$ per $24 \mathrm{~h}$, Figure $7 \mathrm{f}$ ) in the rats with Thy- $1 \mathrm{~N}$ at 7 days. Taken together, the findings suggest that knockdown of $p 300, I R F-1$ and $X A F 1$ genes respectively could not only reduce GMC apoptosis, but also suppress the secondary GMC proliferation and ameliorate the renal function of Thy- $1 \mathrm{~N}$ rats.

\section{Discussion}

In the process of rat Thy-1N, GMCs exhibited different pathological changes including apoptosis, necrosis and proliferation. ${ }^{11,12,14}$ There is growing interest in GMC apoptosis as a potential contributor to the renal lesion. ${ }^{10,14,41,42}$ Early research demonstrated that administration of cobra venom factor (CVF) to deplete complement significantly attenuates renal injury in Thy- $1 \mathrm{~N}$ rats, ${ }^{10,16}$ indicating that GMC apoptosis in rat Thy- $1 \mathrm{~N}$ is complement dependent. Our previous studies have revealed that although C5b-9 deposits were found on the membrane of glomerular cells in Thy- $1 \mathrm{~N}$ rats, many glomerular cells deposited with C5b-9 still remained intact, indicating that these $C 5 b-9$ complexes are sublytic. ${ }^{10,43}$ Our present studies also found that a portion of C5b-9-bound GMCs underwent apoptosis, whereas other C5b-9-bound GMCs did not. These data provide evidences that sublytic C5b-9 is able to trigger GMC apoptosis in the early stage of rat Thy- $1 \mathrm{~N}$.

It has been reported that sublytic $\mathrm{C} 5 \mathrm{~b}-\mathrm{9}$ is able to induce cell apoptosis in many types of cells; ${ }^{20,44,45}$ however, sublytic C5b-9 can protect oligodendrocytes from apoptosis and lead to survival, ${ }^{46}$ suggesting that the different response to $\mathrm{C} 5 \mathrm{~b}-9$ in these cells may be because of different cell types and different biochemical reactions upon sublytic C5b-9 attack. It is worth mentioning that sublytic C5b-9 was also found to be able to induce GMC proliferation at 36 and $54 \mathrm{~h}$ after sublytic C5b-9 stimulation in our previous studies. ${ }^{12}$ Similarly, our present studies revealed that a portion of C5b-9-bound GMCs underwent apoptosis, whereas other C5b-9-bound GMCs could not be found apoptotic. Further studies need to be done to explore the relationship between GMC apoptosis and proliferation due to sublytic C5b-9 and the underlying mechanisms.

Cellular apoptosis can be regulated by the expression of apoptosis-related gene. Our current results manifested that XAF1 expression at the protein level could be induced both in vivo and in vitro. Furthermore, overexpression of XAF1 induced GMC apoptosis, whereas silencing of XAF1 reduced GMC apoptosis in response to sublytic C5b-9 attack, providing evidence that XAF1 might play an important role in the GMC apoptosis triggered by sublytic C5b-9 stimulation. Our previous studies also revealed that IRF-1 induction was related to the GMC apoptosis triggered by sublytic C5b-9. ${ }^{14}$ Wang et al. ${ }^{27}$ reported that JNK1 stimulated XAF1 promoter activity through IRF-1 expression in gastrointestinal cancer in which IRF-1 bound to IRF-E within XAF1 promoter. As a consequence, the roles of IRF-1 in promoting $X A F 1$ gene activation in the GMCs exposed to sublytic C5b-9 were further explored. The results displayed that sublytic C5b-9 could upregulate $X A F 1$ gene expression and increase GMC apoptosis partially via increasing IRF-1 expression. Subsequently, an IRF-1-binding site (IRF-E element) was found to exist in the -65 to $-53 \mathrm{nt}$ region at the $5^{\prime}$ end of XAF1 exon-1, by which IRF-1 could directly bind to XAF1 gene promoter and further activate $X A F 1$ gene. It is interesting to note that the truncated XAF1 promoter $(-337$ to $+160 \mathrm{nt})$ showed higher activity compared with the truncated XAF1 promoter ( -554 to $+160 \mathrm{nt})$, suggesting a negative control element in this truncated region $(-554$ to $-337 \mathrm{nt})$. Further studies need to be done to confirm the possible negative control element and its relationship with IRF-1. Moreover, the truncated XAF promoter $(-14$ to $+160 \mathrm{nt})$ did decrease luciferase activity relative to the truncated XAF promoter ( -47 to $+160 \mathrm{nt})$ in sublytic C5b-9-induced GMCs, indicating there might be a positive control element in this section. However, the truncated XAF promoter $(-14$ to $+160 \mathrm{nt})$ exhibited the same luciferase activity relative to the truncated XAF promoter ( -47 to $+160 \mathrm{nt})$ in GMCs transfected with pcDNA3.1/IRF-1, demonstrating the above-mentioned possible positive control element is not an IRF-1-binding site.

Protein acetylation is an important posttranslational modification that regulates a range of cellular processes. It has been reported IRF-1 can undergo acetylation that might be related to the regulation of IRF-1 activation. ${ }^{32,33}$ Our study revealed that the acetylation of IRF-1 progressed in a time-dependent manner in the GMCs upon sublytic C5b-9 attack. Recently, several studies have reported that all of CBP, p300 and PCAF, as an acetyltransferase, can promote protein acetylation. ${ }^{34,40,47}$ Hence, the interaction between these acetyltransferases and IRF-1 at the protein level was determined, and the results demonstrated that only p300 was able to interact with IRF-1 in sublytic C5b-9-stimulated GMCs.

Multiple evidences support that p300 as a transcription coactivator interacts with numerous transcription factors, and then these proteins act synergistically to increase the expression of their target genes. ${ }^{48,49}$ Our current studies showed that the complex of $\mathrm{p} 300$ and IRF-1 could bind to the $X A F 1$ gene promotor through IRF-1 binding to its element $(-65$ to $-53 \mathrm{nt})$. Subsequent analysis found that p300 gene silence could suppress IRF-1 acetylation and the binding of IRF-1 to XAF1 gene promotor as well as subsequent XAF1 gene transcription and GMC apoptosis due to sublytic C5b-9 stimulation, suggesting that p300 is essential for IRF-1 acetylation and subsequent increase of IRF-1 activity as a transcription factor. Moreover, two acetylation sites of IRF-1 protein (Lys-39 and Lys-78) within the DNA-binding domain were also revealed. Site mutation of Lys-39 and Lys-78 respectively could reduce IRF-1 acetylation and the binding of IRF-1 to XAF1 gene promotor and further suppress XAF1 expression and GMC apoptosis upon sublytic C5b-9 attack. One acetylation site of IRF-1 protein (Lys-78) has been reported by Qi et al., ${ }^{33}$ whereas another acetylation site of IRF-1 protein (Lys-39) is a new one that has not been reported. 
Besides the studies in vitro, our experiments in vivo manifested that blockade of p300, IRF-1 and XAF1 gene expression inhibited GMC apoptosis and secondary GMC proliferation, as well as urinary protein secretion in the rats with Thy-1N. Meanwhile, p300 gene knockdown not only suppressed IRF-1 acetylation, but also reduced XAF1 expression, and IRF-1 gene knockdown also inhibited XAF1 production in the renal tissues of Thy- $1 \mathrm{~N}$ rats, in agreement with our findings in vitro, indicating that $\mathrm{p} 300$-mediated IRF-1dependent XAF1 gene activation was necessary for the development of Thy- $1 \mathrm{~N}$. It is worth mentioning that GMC apoptosis induced by sublytic C5b-9 was also found to be associated with the expression of other genes such as

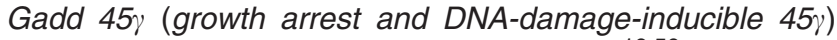
and ATF3 (activating transcription factor 3). ${ }^{10,50}$ Therefore, GMC apoptosis upon sublytic C5b-9 stimulation in Thy-1N rats might require synergetic activation of these apoptosisrelated genes. Further studies need to be carried out to determine the relationship among these genes.

In summary, in the present studies, the roles of $p 300, I R F-1$ and $X A F 1$ genes in GMC apoptosis as well as their relationships and synergistic regulation were examined both in the GMCs triggered by sublytic C5b-9 (in vitro) and in the renal tissues of Thy- $1 \mathrm{~N}$ rats (in vivo). The results indicated that sublytic C5b-9, as a trigger for GMC lesions, could lead to GMC apoptosis of Thy- $1 \mathrm{~N}$ rats through upregulating these genes. In the process, sublytic C5b-9 could induce p300 and IRF-1 expression simultaneously. The IRF-1 activation of $X A F 1$ gene and its regulation via p300-dependent IRF-1 acetylation might play a vital role in mediating GMC apoptosis.

\footnotetext{
Materials and Methods

Reagents and animals. Monoclonal antibodies (Abs) against IRF-1 (sc-640), C5b-9 (sc-58935), His (sc-53073) and Thy-1 antigen (OX-7, sc-53116) as well as polyclonal Abs against PCAF (sc-8999) were purchased from Santa Cruz Biotechnology (Santa Cruz, CA, USA). Polyclonal Abs against XAF1 (ab17204) and CBP (ab32646) and a monoclonal Ab against p300 (ab3164) were purchased from Abcam (Cambridge, UK). Monoclonal Abs against acetylated-Lysine (9681), K63-linkage specific polyubiquitin (5621) and $\beta$-actin (3700) and polyclonal Abs against K48-linkage specific polyubiquitin (4289) as well as HRP-conjugated antirabbit (7074) and anti-mouse IgG (7076) as well as anti-rabbit IgG (Conformation Specific, 5127) were purchased from Cell Signaling Technology (Danvers, MA, USA). Cy5-linked anti-mouse IgG (115-175-071) was from Jackson ImmunoResearch Laboratories (West Grove, PA, USA). Enhanced chemiluminescence $(E C L)$ western blotting substrate and co-IP kit and RIPA lysis buffer were supplied by Thermo (Fremont, CA, USA). The ChIP assay kit was from Millipore (Bedford, MA, USA). The vector of pcDNA3.1, Lipofectamine 2000 and Neon transfection system were purchased from Invitrogen (Carlsbad, CA, USA). The incision enzymes BamHI, Hindlll, Kpnl and T4 DNA were from NEB (Ipswich, Suffolk, UK). The expression plasmids of pEGFP-N1 were supplied by Clontech (Mountain View, CA, USA). pGL3-basic vector and pRL-SV40 luciferase reporter were purchased from Promega (Madison, WI, USA). The shRNA expression plasmids of pGCsi.U6.neo.GFP were from Genechem (Shanghai, China). A site-directed mutagenesis kit was from Agilent Technologies-Stratagene (Santa Clara, CA, USA). QIAprep spin miniprep kit was obtained from Biomiga (San Diego, CA, USA). An annexin V-APC/ PI kit was purchased from Bender MedSystems (Vienna, Austria). A TUNEL staining kit was from Roche (Basel, Switzerland). SD rats were from B\&K Universal Ltd (Shanghai, China). An institutional approval for the animal study protocol was obtained. Rat GMC and 293T were provided by China Centre for Type Culture Collection (Wuhan, China) and American Tissue Culture Collection (Manassas, VA, USA), respectively. Normal serum (NS) from 10 healthy adult donors and HIS was obtained by incubating NS at $56^{\circ} \mathrm{C}$ for $30 \mathrm{~min}$. Human complement C6-deficient serum (C6DS) was obtained from Sigma (St. Louis, MO, USA). Purified human
}

complement C6 was from Sino Biological Inc. (Beijing, China). Rabbit polyclonal Abs against Thy-1 antigen (anti-Thy-1 Ab) were prepared according to previously published procedures. ${ }^{43,51}$

Plasmid construction. The plasmids of pEGFP-N1/XAF1 (pXAF1) were constructed by inserting the ORF of rat XAF1 (XM_001053682) CDNA into pEGFP-N1. The XAF1 gene was amplified by polymerase chain reaction (PCR) from CDNA of rat GMCs. The PCR products and pcDNA3.1 vector were digested with BamHI and Hindlll, and then ligated using T4 DNA ligase. Here, rattus IRF-1 (NM_012591.1) expression plasmids (pcDNA3.1/IRF-1) have been constructed in our previous study. ${ }^{14}$ His-tag was added to the $3^{\prime}$ of IRF-1 by PCR to obtain pcDNA3.1/IRF-1-His. IRF-1 mutants (C29A, C39A, C50A, C66A, C70A, C75A and $\mathrm{C} 78 \mathrm{~A}$ ) were generated by using a site-directed mutagenesis kit according to the manufacturer's procedures with above-mentioned wild-type pcDNA3.1/IRF-1-His as the template.

shRNA generation. To silence rat $p 300$ and $X A F 1$ genes, different shRNA sequences against the mRNA of p300 (XM_001076610) and XAF1 (XM_001053682) were designed. The plasmids of p300 shRNA (shp300) and XAF1 shRNA (shXAF1) were constructed by using pGCsi.U6.neo.GFP, and the most effective shRNA expression plasmids were chosen for further experiments. Meanwhile, scrambled control shRNA (shCTR) expression plasmids were produced as a negative control. Here, rattus IRF-1 (NM_012591.1) shRNA expression plasmids (shIRF-1) have been constructed in our previous study. ${ }^{14}$

Lentiviral shRNA packing. Lentiviral p300 shRNA (Lv-shp300), lentiviral IRF-1 shRNA (Lv-shIRF-1), lentiviral XAF1 shRNA (Lv-shXAF1) and lentiviral control shRNA (Lv-shCTR) were purchased from GenePharma (Shanghai, China). ${ }^{52,53}$ The shRNA oligonucleotide sequences used to knock down the expression of p300, IRF-1 and XAF1 genes are the same as the above-mentioned most effective shRNAs against these genes as follows: shp300, $5^{\prime}$-CAGCTCTATA GAGCGAGCTTA-3'; shIRF-1, 5'-CAGCTCTACTCTGCCTGATGA-3'; shXAF1, $5^{\prime}$-CAGCAGACCAAGGAAAGCCAA-3'; and shCTR, 5'-TTCTCCGAACGTGTCA CGT-3'.

XAF1 promoter luciferase reporter construction. The pGL3-XAF1full-length (pGL3-XAF1-FL) was constructed by inserting the $1.657 \mathrm{~kb}$ rat XAF1 promoter ( -1496 to $+160 \mathrm{nt})$ into pGL3-basic vector. This construct contains nucleotides 58, 599, $855-58,601,510$ from the reference genomic sequence NC_005109.2. To determine the minimal XAF1 promoter sequence required for constitutive and inducible activity, we constructed the following promoter deletion fragments by PCR and cloned them into the same reporter vector: -554 to $+160,-337$ to $+160,-47$ to +160 and -14 to +160 nt. Specific primers for full-length and different deletion fragments are listed in Supplementary Table S1. pGL3-XAF1 mutant (ACTTTCGGTTTTG $\rightarrow$ ACTCTCAGTCTTG, pGL3$X A F 1^{M}$ ) was also generated using a site-directed mutagenesis kit according to the manufacturer's procedures with above-mentioned wild-type pGL3-XAF1 full-length (pGL3-XAF1-FL ${ }^{\mathrm{WT}}$ ) as the template.

Cell culture and sublytic C5b-9 determination. Rat GMCs were cultured in MEM as previously described. ${ }^{10}$ The concentrations of Thy- $1 \mathrm{Ab}$ and complement were $5 \%$ Thy $-1 \mathrm{Ab}$ and $4 \%$ NHS respectively, with $<5 \%$ lactate dehydrogenase $(\mathrm{LDH})$ release (Supplementary Figure S11A and B) as previously reported. ${ }^{10,54,55}$ To demonstrate whether the response was due to sublytic C $5 \mathrm{~b}-9$ ( $5 \%$ Thy-1 Ab $+4 \%$ NHS), GMCs were treated with $5 \%$ Thy-1 Ab, 5\% Thy-1 Ab + 4\% HIS, $5 \%$ Thy $-1 \mathrm{Ab}+4 \%$ C6DS, $5 \%$ Thy $-1 \mathrm{Ab}+4 \%$ C6DS + C6 (0.5 or $2 \mathrm{mg} / \mathrm{l})$ and $5 \%$ Thy $-1 \mathrm{Ab}+4 \%$ C6DS + PBS. ${ }^{10,55,56}$ Adding C6 back to C6DS could recover its ability to induce LDH release from GMCs (Supplementary Figure S11C). Moreover, $293 \mathrm{~T}$ cells were cultured in Dulbecco's MEM (DMEM) supplemented with 10\% FBS.

Cell transfection and identification. GMCs were transfected with corresponding plasmids by using Neon transfection system. ${ }^{12,57}$ The 293T cells were transfected using Lipofectamine 2000 according to the manufacturer's instructions. ${ }^{57}$ The transfection efficiency was examined by the fluorescence of green fluorescence protein (GFP; Supplementary Figure S12) or the expression of corresponding protein (Supplementary Figure S13).

Thy-1N model reproduction and experimental design. First, male SD rats $(180-200 \mathrm{~g})$ were randomly divided into two groups $(n=6$ in each time 
point/group). (1) Thy-1N group: rats were given Thy-1 Ab $(0.75 \mathrm{ml}$ per $100 \mathrm{~g})$ by a single i.p. injection. (2) NS group: rats were injected i.p. with normal rabbit serum $(0.75 \mathrm{ml}$ per $100 \mathrm{~g})$. Rat renal cortexes were obtained by killing at $0,1,2,3,6$ and $12 \mathrm{~h}$, and were then examined for the expression of $\mathrm{p300}$, IRF-1 and XAF1, the interaction between $\mathrm{p} 300$ and IRF-1, as well as the acetylation of IRF-1 by immunoblot (IB) and co-IP.

To confirm the roles of p300, IRF-1 and XAF1 in apoptotic and proliferative changes of Thy- $1 \mathrm{~N}$ rats, male SD rats $(180-200 \mathrm{~g})$ were divided into six groups ( $n=6-8$ in each time point/group), namely: (1) NS, (2) Thy-1N, (3) Lv-shp300 + Thy-1N, (4) Lv-shIRF-1 + Thy-1N, (5) Lv-shXAF1 + Thy-1N and (6) Lv-shCTR + Thy- $1 \mathrm{~N}$. The rats belonging to NS and Thy- $1 \mathrm{~N}$ groups were treated with the abovementioned methods. For 3-6 groups, the lentiviral shRNA (Lv-shRNA) was transfected into rat kidney via renal artery perfusion followed by renal veins occlusion for 10 min immediately. After 4 days, Thy- $1 \mathrm{~N}$ was induced as mentioned above. Rat renal cortexes were then collected by killing at $3 \mathrm{~h}$ and $6 \mathrm{~h}$ and on day 7 after Thy-1 Ab or NS injection. GFP expression was observed to define the efficiency of transferring Lv-shRNA into rat kidneys (Supplementary Figure S14), and the efficiency of gene silencing was evaluated by IB assay. The pathological changes of GMC were determined by light microscopy (LM) and EM. Parts of renal tissues were also examined by using IB and co-IP analysis for the expression of corresponding genes including their interaction at protein level.

IB analysis. Cultured GMCs or 293T and rat renal tissues were lysed using RIPA lysis buffer. Equal amounts ( $40 \mu \mathrm{g} / \mathrm{lane}$ ) of protein were subjected to SDS-PAGE gel respectively. IB analysis was performed as described previously. ${ }^{10}$ $\beta$-Actin was used as an internal control of protein loading and the relative protein level in each group was expressed relative to control group. Each sample was assayed in triplicate.

Co-IP experiment. A total of $400 \mu \mathrm{g}$ of extract prepared from GMCs, 293T or rat renal tissues was mixed with $40 \mu \mathrm{l}$ protein G-Sepharose beads in co-IP assay buffer, incubated for $2 \mathrm{~h}$ and centrifuged for $2 \mathrm{~min}$. The recovered supernatant was incubated with the corresponding $\mathrm{Abs}(2 \mathrm{~g}$, preimmune $\mathrm{Ig} \mathrm{G}$ as a control reaction) at $4^{\circ} \mathrm{C}$ overnight. Then, $40 \mu \mathrm{l}$ of protein G-Sepharose beads was added, and the incubation was continued for $2 \mathrm{~h}$. Protein G-precipitated protein complex was recovered by centrifugation and harvested beads resuspended in $30 \mu \mathrm{l}$ of $2 \times$ SDS PAGE sample buffer were boiled for $5 \mathrm{~min}$. The samples were then analyzed by IB assay with specific Abs. A $40 \mu \mathrm{g}$ aliquot of whole-cell extract (WCE) was used as an input control.

Flow cytometry. $5 \times 10^{5}$ GMCs were resuspended in binding buffer containing Annexin V-APC and propidium iodide. ${ }^{58}$ The samples were analyzed on a FACScan flow cytometer (BD, Franklin Lakes, NJ, USA). The percentage of apoptotic cells in a 10000 cell cohort was determined by flow cytometry. TUNEL staining (TMR-labeled) was used to label the cells $\left(5 \times 10^{5} \mathrm{GMCs}\right)$ at $6 \mathrm{~h}$ after sublytic C5b-9 stimulation. Subsequently, flow cytometry analysis was performed to detect the cellular apoptosis. ${ }^{41}$ In addition, both TUNEL staining (FITC-labeled) and the Ab against C5b-9 (adding Cy3-labeled secondary Ab) were used to label the cells $\left(5 \times 10^{5} \mathrm{GMCs}\right)$ at $6 \mathrm{~h}$ after sublytic C5b-9 stimulation. Flow cytometry analysis was then performed to detect the cellular apoptosis and C5b-9 deposits on GMC membrane.

Luciferase reporter assay. The activity of full-length, different deletion fragments and mutant XAF1 gene promotor in the GMCs stimulated with sublytic C5b-9 or overexpression of IRF-1 was detected by luciferase reporter assay. The luciferase activity was measured as mentioned previously. ${ }^{14}$

ChIP and re-ChIP assays. ChIP was done by using Abs against IRF-1 and His, and preimmune mouse $\operatorname{lgG}$, respectively, as mentioned previously. ${ }^{14}$ A proximal region in XAF1 promotor ( -169 to -19 nt) was amplified from the immunoprecipitated chromatin by PCR using a pair of primers (sense, $5^{\prime}$-GAATGG CCTTCTGGGAGTAT-3' and antisense, $5^{\prime}$-CTTCGGTGGAGTTTCTGTTT-3'). Re-ChIP was performed by using an $A b$ against p300 and preimmune mouse IgG respectively. The proximal region in XAF1 gene promotor $(-169$ to $-19 \mathrm{nt})$ was amplified from the products of re-ChIP through PCR by using the above-mentioned primers, and the results were normalized to input. ${ }^{59}$

Renal histological examination. For LM, paraffin-embedded renal histological sections $(4 \mu \mathrm{m})$ of rats on day 7 after nephritis induction were stained with $\mathrm{H} \& \mathrm{E}$, and 100 glomerular cross-sections from each rat were examined according to the same method. ${ }^{15}$ For EM, ultrathin sections of renal tissues were stained with uranyl acetate and lead citrate, and the ultrastructural changes were observed. ${ }^{51}$

Immunofluorescence (IF) staining. To detect GMC proliferation, frozen sections $(4 \mu \mathrm{m})$ of rat renal tissues were stained with an $A b$ to Thy- 1 antigen (OX7 , as a marker of GMCs) ${ }^{60}$ Then, the sections were incubated with corresponding Cy5-conjugated secondary $\mathrm{Ab}$. In addition, the number of glomerular OX-7positive cells was counted in a double-blinded manner by two independent observers counting 100 glomerular cross-sections from each rat as described previously. ${ }^{15}$

TUNEL analysis in vivo. Frozen sections $(4 \mu \mathrm{m})$ of rat renal tissues were treated with proteinase $\mathrm{K}$, and incubated with $50 \mu$ reaction mixture of TUNEL for $60 \mathrm{~min}$. The number of TUNEL-positive nuclei in 100 glomerular cross-sections was counted in a double-blinded manner under fluorescence microscopy. ${ }^{10,15}$

Urine protein detection. Rat urine in different groups was collected on day 7 after the corresponding treatments. The contents of urinary protein (mg per $24 \mathrm{~h}$ ) were measured by the total protein UC FS (DiaSys Diagnostic Systems, Holzheim, Germany). Each sample was assayed in triplicate.

Statistical analysis. Data are presented as means \pm S.E. One-way ANOVA was used to determine significant differences among groups. Where significant differences were found, individual comparisons were made between groups using the $t$-statistic and adjusting the critical value according to the Bonferroni method. $P<0.05$ was considered significant.

\section{Conflict of Interest}

The authors declare no conflict of interest.

Acknowledgements. The study was supported by grants from National Natural Science Foundations of China (nos. 31000396, 81072402 and 81273333) and grants from Natural Science Foundations of Jiangsu Province in China (BK2009417, 10KJB310006, 09hx43). The study was also supported by a grant from A Project Funded by the Priority Academic Program Development of Jiangsu Higher Education Institutions (PAPD) and a grant from the Technology Development Foundation of Nanjing Medical University (2013NJMU232). We also thank Dr. Feng Lin (Cleveland Clinic, USA) for reviewing the manuscript.

\section{Author contributions}

Yingwei Wang and Wen Qiu designed the study and wrote the manuscript. Wen Qiu, Jianbo Zhou and Ganqian Zhu conceived and carried out experiments and analyzed data. Dan Zhao, Fengxia He, Jing Zhang, Yanlai Lu, Tianyi Yu and Lisha Liu participated in the experiments. All authors had final approval of the submitted and published versions.

1. Tumlin JA, Madaio MP, Hennigar R. Idiopathic IgA nephropathy: pathogenesis, histopathology, and therapeutic options. Clin J Am Soc Nephrol 2007; 2: 1054-1061.

2. Tamouza H, Chemouny JM, Raskova Kafkova L, Berthelot L, Flamant M, Demion M et al. The IgA1 immune complex-mediated activation of the MAPK/ERK kinase pathway in mesangial cells is associated with glomerular damage in IgA nephropathy. Kidney Int 2012; 82: 1284-1296.

3. Nagai K, Miyoshi M, Kake T, Fukushima N, Matsuura M, Shibata E et al. Dual involvement of growth arrest-specific gene 6 in the early phase of human IgA nephropathy. PLoS One 2013; 8: e66759.

4. Chihara Y, Ono H, Ishimitsu T, Ono Y, Ishikawa K, Rakugi H et al. Roles of TGF-beta1 and apoptosis in the progression of glomerulosclerosis in human IgA nephropathy. Clin Nephrol 2006; 65: 385-392.

5. Espinosa M, Ortega R, Gomez-Carrasco JM, Lopez-Rubio F, Lopez-Andreu M, Lopez-Oliva MO et al. Mesangial C4d deposition: a new prognostic factor in IgA nephropathy. Nephrol Dial Transplant 2009; 24: 886-891.

6. Stangou M, Alexopoulos E, Pantzaki A, Leonstini M, Memmos D. C5b-9 glomerular deposition and tubular alpha3beta1-integrin expression are implicated in the development of chronic lesions and predict renal function outcome in immunoglobulin A nephropathy. Scand J Urol Nephrol 2008; 42: 373-380. 
7. Onda K, Ohsawa I, Ohi H, Tamano M, Mano S, Wakabayashi M et al. Excretion of complement proteins and its activation marker C5b-9 in IgA nephropathy in relation to renal function. BMC Nephrol 2011; 12: 64.

8. Cina DP, Xu H, Liu L, Farkas L, Farkas D, Kolb $M$ et al. Renal tubular angiogenic dysregulation in anti-Thy1.1 glomerulonephritis. Am J Physiol 2011; 300: F488-F498.

9. Liu X, Lv Y, Xie Y, Hong Q, Cai G, Zhang S et al. Change of MAX interactor 1 expression in an anti-Thy1 nephritis model and its effect on mesangial cell proliferation. Cell Physiol Biochem 2011; 27: 391-400.

10. Qiu W, Che N, Feng X, Xia M, Wang H, Zhao D et al. Apoptosis of glomerular mesangial cells induced by sublytic C5b-9 complexes in rats with Thy-1 nephritis is dependent on Gadd45gamma upregulation. Eur J Immunol 2009; 39: 3251-3266.

11. Shimizu A, Masuda $Y$, Kitamura $H$, Ishizaki M, Ohashi R, Sugisaki $Y$ et al. Complementmediated killing of mesangial cells in experimental glomerulonephritis: cell death by a combination of apoptosis and necrosis. Nephron 2000; 86: 152-160.

12. Qiu W, Zhang Y, Liu X, Zhou J, Li Y, Zhou $Y$ et al. Sublytic C5b-9 complexes induce proliferative changes of glomerular mesangial cells in rat Thy-1 nephritis through TRAF6-mediated PI3K-dependent Akt1 activation. J Pathol 2012; 226: 619-632.

13. Mima A, Abe H, Nagai K, Arai H, Matsubara T, Araki M et al. Activation of Src mediates PDGF-induced Smad1 phosphorylation and contributes to the progression of glomerulosclerosis in glomerulonephritis. PloS One 2011; 6: e17929.

14. Liu L, Qiu W, Wang H, Li Y, Zhou J, Xia M et al. Sublytic C5b-9 complexes induce apoptosis of glomerular mesangial cells in rats with Thy-1 nephritis through role of interferon regulatory factor-1-dependent caspase 8 activation. J Biol Chem 2012; 287: 16410-16423.

15. Sato T, Van Dixhoorn MG, Prins FA, Mooney A, Verhagen N, Muizert Y et al. The terminal sequence of complement plays an essential role in antibody-mediated renal cell apoptosis. J Am Soc Nephrol 1999; 10: 1242-1252.

16. Yamamoto T, Wilson CB. Complement dependence of antibody-induced mesangial cell injury in the rat. $\mathrm{J}$ Immunol 1987; 138: 3758-3765.

17. Fosbrink M, Cudrici C, Tegla CA, Soloviova K, Ito T, Vlaicu $S$ et al. Response gene to complement 32 is required for C5b-9 induced cell cycle activation in endothelial cells. Exp Mol Pathol 2009; 86: 87-94.

18. Kunchithapautham K, Bandyopadhyay M, Dahrouj M, Thurman JM, Rohrer B. Sublytic membrane-attack-complex activation and VEGF secretion in retinal pigment epithelial cells. Adv Exp Med Biol 2012; 723: 23-30.

19. Triantafilou K, Hughes TR, Triantafilou M, Morgan BP. The complement membrane attack complex triggers intracellular $\mathrm{Ca} 2$ + fluxes leading to NLRP3 inflammasome activation. J Cell Sci 2013; 126(Pt 13): 2903-2913.

20. Ren G, Huynh C, Bijian K, Cybulsky AV. Role of apoptosis signal-regulating kinase 1 in complement-mediated glomerular epithelial cell injury. Mol Immunol 2008; 45: 2236-2246.

21. Niculescu F, Rus H, van Biesen T, Shin ML. Activation of Ras and mitogen-activated protein kinase pathway by terminal complement complexes is $\mathrm{G}$ protein dependent. J Immunol 1997; 158: 4405-4412.

22. Zou B, Chim CS, Pang R, Zeng H, Dai Y, Zhang R et al. XIAP-associated factor 1 (XAF1), a novel target of $\mathrm{p} 53$, enhances $\mathrm{p53}$-mediated apoptosis via post-translational modification. Mol Carcinog 2012; 51: 422-432.

23. Tu SP, Sun YW, Cui JT, Zou B, Lin MC, Gu Q et al. Tumor suppressor XIAP-Associated factor 1 (XAF1) cooperates with tumor necrosis factor-related apoptosis-inducing ligand to suppress colon cancer growth and trigger tumor regression. Cancer 2010; 116: $1252-1263$

24. Chen $X Y, H e Q Y$, Guo MZ. XAF1 is frequently methylated in human esophageal cancer. World J Gastroenterol 2012; 18: 2844-2849.

25. Wang $Y$, Mao $H, H a o ~ Q$, Wang $Y, Y a n g ~ Y$, Shen $L$ et al. Association of expression of XIAP-associated factor 1 (XAF1) with clinicopathologic factors, overall survival, microvessel density and cisplatin-resistance in ovarian cancer. Regul Pept 2012; 178: $36-42$.

26. Gao J, Senthil M, Ren B, Yan J, Xing Q, Yu J et al. IRF-1 transcriptionally upregulates PUMA, which mediates the mitochondrial apoptotic pathway in IRF-1-induced apoptosis in cancer cells. Cell Death Differ 2010; 17: 699-709.

27. Wang J, Zhang W, Zhang Y, Chen Y, Zou B, Jiang B et al. c-Jun N-terminal kinase (JNK1) upregulates XIAP-associated factor 1 (XAF1) through interferon regulatory factor 1 (IRF-1) in gastrointestinal cancer. Carcinogenesis 2009; 30: 222-229.

28. Pejanovic N, Hochrainer K, Liu T, Aerne BL, Soares MP, Anrather J. Regulation of nuclear factor kappaB (NF-kappaB) transcriptional activity via p65 acetylation by the chaperonin containing TCP1 (CCT). PLoS One 2012; 7: e42020.

29. Kim DH, Kim JM, Lee EK, Choi YJ, Kim CH, Choi JS et al. Modulation of FoxO1 phosphorylation/acetylation by baicalin during aging. J Nutr Biochem 2011; 23: 1277-1284.

30. Dornan D, Eckert M, Wallace M, Shimizu H, Ramsay E, Hupp TR et al. Interferon regulatory factor 1 binding to $\mathrm{p} 300$ stimulates DNA-dependent acetylation of p53. Mol Cell Biol 2004; 24: 10083-10098.

31. Dhupar R, Klune JR, Evankovich J, Cardinal J, Zhang M, Ross M et al. Interferon regulatory factor 1 mediates acetylation and release of high mobility group box 1 from hepatocytes during murine liver ischemia-reperfusion injury. Shock 2011; 35 : 293-301.

32. Masumi A, Ozato K. Coactivator p300 acetylates the interferon regulatory factor-2 in U937 cells following phorbol ester treatment. J Biol Chem 2001; 276: 20973-20980.

33. Qi H, Zhu H, Lou M, Fan Y, Liu H, Shen J et al. Interferon regulatory factor 1 transactivates expression of human DNA polymerase eta in response to carcinogen N-methyl-N'-nitro-Nnitrosoguanidine. J Biol Chem 2012; 287: 12622-12633.

34. Holmqvist PH, Boija A, Philip P, Crona F, Stenberg P, Mannervik M. Preferential genome targeting of the CBP co-activator by Rel and Smad proteins in early Drosophila melanogaster embryos. PLoS Genet 2012; 8: e1002769.

35. Sakamoto S, Wakae K, Anzai Y, Murai K, Tamaki N, Miyazaki M et al. E2A and CBP/p300 act in synergy to promote chromatin accessibility of the immunoglobulin kappa locus. $J$ Immunol 2012; 188: 5547-5560.

36. Jain AK, Allton K, lacovino M, Mahen E, Milczarek RJ, Zwaka TP et al. p53 regulates cell cycle and microRNAs to promote differentiation of human embryonic stem cells. PLOS Biol 2012; 10: e1001268.

37. Cheng SE, Lin CC, Lee IT, Hsu CK, Kou YR, Yang CM. Cigarette smoke extract regulates cytosolic phospholipase A2 expression via NADPH oxidase/MAPKs/AP-1 and p300 in human tracheal smooth muscle cells. J Cell Biochem 2011; 112: 589-599.

38. Chen R, Xu M, Hogg RT, Li J, Little B, Gerard RD et al. The acetylase/deacetylase couple creb binding protein/sirtuin 1 controls hypoxia inducible factor 2 signaling. J Biol Chem 2012; 287: 30800-30811.

39. Ge X, Jin Q, Zhang F, Yan T, Zhai Q. PCAF acetylates \{beta\}-catenin and improves its stability. Mol Biol Cell 2009; 20: 419-427.

40. Li Y, Li C, Sun L, Chu G, Li J, Chen F et al. Role of p300 in regulating neuronal nitric oxide synthase gene expression through nuclear factor-kappaB-mediated way in neuronal cells. Neuroscience 2013; 248: 681-689.

41. He D, Li J, Zhao J, Fei J, Zhang X. C/EBP homologous protein induces mesangial cell apoptosis under hyperglycemia. Mol Med Rep 2013; 7: 445-448.

42. Chen YQ, Wang XX, Yao XM, Zhang DL, Yang XF, Tian SF et al. Abated microRNA-195 expression protected mesangial cells from apoptosis in early diabetic renal injury in mice. $J$ Nephrol 2012; 25: 566-576.

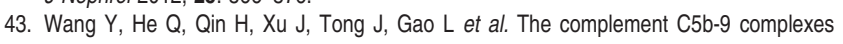
induced injury of glomerular mesangial cells in rats with Thy-1 nephritis by increasing nitric oxide synthesis. Life Sci 2006; 79: 182-192.

44. Magro CM, Wang X. Cocaine-associated retiform purpura: a c5b-9-mediated microangiopathy syndrome associated with enhanced apoptosis and high levels of intercellular adhesion molecule-1 expression. Am J Dermatopathol 2013; 35: 722-730.

45. Jha P, Banda H, Tytarenko R, Bora PS, Bora NS. Complement mediated apoptosis leads to the loss of retinal ganglion cells in animal model of glaucoma. Mol Immunol 2011; 48: 2151-2158.

46. Cudrici C, Niculescu F, Jensen T, Zafranskaia E, Fosbrink M, Rus V et al. C5b-9 terminal complex protects oligodendrocytes from apoptotic cell death by inhibiting caspase-8 processing and up-regulating FLIP. J Immunol 2006; 176: 3173-3180.

47. Love IM, Sekaric P, Shi D, Grossman SR, Androphy EJ. The histone acetyltransferase PCAF regulates p21 transcription through stress-induced acetylation of histone $\mathrm{H} 3$. Cell Cycle 2012; 11: 2458-2466.

48. Mukherjee SP, Behar M, Birnbaum HA, Hoffmann A, Wright PE, Ghosh G. Analysis of the RelA:CBP/p300 interaction reveals its involvement in NF-kappaB-driven transcription. PLOS Biol 2013; 11: e1001647.

49. Sengupta S, Mantha AK, Mitra S, Bhakat KK. Human AP endonuclease (APE1/Ref-1) and its acetylation regulate YB-1-p300 recruitment and RNA polymerase II loading in the drug-induced activation of multidrug resistance gene MDR1. Oncogene 2011; 30: 482-493.

50. Xu K, Zhou Y, Qiu W, Liu X, Xia M, Liu L et al. Activating transcription factor 3 (ATF3) promotes sublytic C5b-9-induced glomerular mesangial cells apoptosis through up-regulation of Gadd45alpha and KLF6 gene expression. Immunobiology 2011; 216: 871-881.

51. Xu JH, Qiu W, Wang YW, Xu J, Tong JX, Gao LJ et al. Gene expression profile and overexpression of apoptosis-related genes (NGFI-B and Gadd 45 gamma) in early phase of Thy-1 nephritis model. Cell Tissue Res 2006; 326: 159-168.

52. Xie W, Feng $Q$, Su Y, Dong B, Wu J, Meng L et al. Transcriptional regulation of PES1 expression by c-Jun in colon cancer. PLoS One 2012; 7: e42253.

53. Zhang Y, Zhang X, Wang X, Gan L, Yu G, Chen Y et al. Inhibition of LDH-A by lentivirusmediated small interfering RNA suppresses intestinal-type gastric cancer tumorigenicity through the downregulation of Oct4. Cancer Lett 2012; 321: 45-54.

54. Cybulsky AV, Takano T, Papillon J, Bijian K, Guillemette J. Activation of the extracellular signal-regulated kinase by complement C5b-9. Am J Physiol 2005; 289: F593-F603.

55. Couser WG, Pippin JW, Shankland SJ. Complement (C5b-9) induces DNA synthesis in rat mesangial cells in vitro. Kidney Int 2001; 59: 905-912.

56. Shankland SJ, Pippin JW, Couser WG. Complement (C5b-9) induces glomerular epithelial cell DNA synthesis but not proliferation in vitro. Kidney Int 1999; 56: 538-548. 
57. Yunus MA, Chung LM, Chaudhry Y, Bailey D, Goodfellow I. Development of an optimized RNA-based murine norovirus reverse genetics system. J Virol Methods 2010; 169 112-118.

58. Juszczak K, Kaszuba-Zwoinska J, Thor PJ. Pulsating electromagnetic field stimulation of urothelial cells induces apoptosis and diminishes necrosis: new insight to magnetic therapy in urology. J Physiol Pharmacol 2012; 63: 397-401.

59. Zhou B, Liu Y, Kahn M, Ann DK, Han A, Wang H et al. Interactions between beta-catenin and transforming growth factor-beta signaling pathways mediate epithelial-mesenchymal transition and are dependent on the transcriptional co-activator CAMP-response elementbinding protein (CREB)-binding protein (CBP). J Biol Chem 2012; 287: 7026-7038.

60. Daniel C, Albrecht H, Ludke A, Hugo C. Nestin expression in repopulating mesangial cells promotes their proliferation. Laboratory Invest 2008; 88: 387-397.
Cell Death and Disease is an open-access journal published by Nature Publishing Group. This work is licensed under a Creative Commons Attribution-NonCommercialShareAlike 3.0 Unported License. The images or other third party material in this article are included in the article's Creative Commons license, unless indicated otherwise in the credit line; if the material is not included under the Creative Commons license, users will need to obtain permission from the license holder to reproduce the material. To view a copy of this license, visit http://creativecommons.org/licenses/ by-nc-sa/3.0/

Supplementary Information accompanies this paper on Cell Death and Disease website (http://www.nature.com/cddis) 